\title{
Assessing the Robustness of Feature-Based Selection in Visual Working Memory
}

\author{
Andra Arnicane ${ }^{1}$ and Alessandra S. Souza ${ }^{1,2}$ \\ ${ }^{1}$ University of Zurich, Switzerland \\ ${ }^{2}$ University of Porto, Portugal
}

This is the accepted version for publication in the

Journal of Experimental Psychology: Human Perception and Performance

(C) 2021, American Psychological Association. This paper is not the copy of record and may not exactly replicate the final, authoritative version of the article. Please do not copy or cite without authors' permission. The final article will be available, upon publication, via its DOI: 10.1037/xhp0000911

\section{Author Note}

Andra Arnicane, Orcid: https://orcid.org/0000-0002-7304-3381

Alessandra S. Souza, Orcid: https://orcid.org/0000-0002-1057-8426

We have no known conflict of interest to disclose. This research was supported by a grant from the Velux Foundation to A. S. Souza (project $\mathrm{n}^{\circ} 1053$ ). The study materials and data are available at Open Science Framework: https://osf.io/r45a6

Correspondence concerning this article should be addressed to Andra Arnicane, Department of Psychology, University of Zurich, Binzmühlestrasse 14, CH-8050, Zürich, Switzerland. E-mail: arnicanea@ hotmail.com 


\begin{abstract}
Visual working memory (VWM) is a capacity-limited system to temporarily maintain visual information. Attending to information in VWM conveys a benefit, as revealed by the retro-cue effect. For example, when the location of one memory item is retro-cued during VWM maintenance, memory accuracy for that item improves. Attentional selection in VWM can also be feature-based: One feature (e.g., shape) may serve as a retrieval cue for another feature (e.g., color) of the same item. Here, we assessed the scope of feature retro-cue benefits with continuous report of colors and orientations. Across six experiments, we observed robust feature retro-cue benefits with manipulations of the cued and recalled feature dimensions, as well as against different baselines controlling for temporal and interference effects. Furthermore, we replicated with continuous report the hallmark of external feature-based attention - concurrent selection of multiple items. Mixture modelling indicated that feature retro-cue benefits increased recall probability and sometimes precision, paralleling findings on spatial attention. Importantly, cueing multiple items did not produce costs, indicating that concurrently attended items did not interfere with each other. Lastly, manipulation constraining spatial location to a single position suggested that feature retro-cue benefits persist even when spatial context is not singular, but take longer to emerge.

Keywords: Visual working memory; attention; feature retro-cue; mixture modeling; continuous features
\end{abstract}




\section{Statement of Public Significance}

Our capacity to keep visual information available in mind is limited. For example, memory gets poorer, the more players or locations we need to follow on a board game. This is because memory items compete with each other, making their retrieval harder. Focused attention allows one to bypass this limitation: We can focus attention to the location where we saw a relevant object, or we can use one of its features (e.g., color) to select it. Here, we demonstrate robust benefits of focusing attention on different visual features (color, orientation, or shape), and this benefit did not depend on number of feature-values used for retrieval and the number of objects selected simultaneously. Feature-based selection is only diminished when objects are perceived in the same spatial location - indicating that features and spatial locations are used conjointly for disambiguating visual objects. 


\section{Assessing the Robustness of Feature-Based Selection in Visual Working Memory}

Visual working memory (VWM) enables ongoing cognition by maintaining and updating visual information, but its capacity is severely limited: On average, only up to four visual items can be concurrently maintained in VWM (Cowan, 2010; Luck \& Vogel, 2013). Capacity limitations arise in part because memory items within VWM interfere with each other (Oberauer \& Lin, 2017; Pertzov et al., 2017). Accordingly, one way to bypass this interference is by efficient selection of the information that is relevant at a given time. To achieve this selective function, an internal focus of attention within VWM has been proposed that prioritizes information: Information in the focus of attention can be retrieved faster and is better protected from interference than other encoded but not focused representations in VWM (Oberauer \& Lin, 2017).

The selective prioritization of a memory item can be examined by guiding attention to one or even a subset of items held in mind during the maintenance phase by presenting retro-cues. Traditionally, the retro-cueing procedure involves the presentation of a cue (e.g., a central arrow) pointing towards the location one of the items occupied in the memory array, signaling that this item is most likely to be tested. Since the introduction of the retro-cue procedure (Griffin \& Nobre, 2003; Landman et al., 2003), ample research demonstrated that retro-cueing spatial locations in VWM yields benefits to accuracy as well as retrieval speed (for a review, see Souza \& Oberauer, 2016).

More recent work has revealed that, additionally to spatial locations, also visual features - e.g., color or shape - can be used for attentional selection in VWM, resulting in retro-cue benefits for memory accuracy and response speed (Gilchrist et al., 2016; Heuer 
\& Schubö, 2016; Kalogeropoulou et al., 2017; Li \& Saiki, 2015; Pertzov et al., 2013). In these studies, the retro-cue presents one feature of the memory item (e.g., its shape), and another feature of this item has to be retrieved (e.g., its color). The conditions that give rise to feature retro-cue benefits, however, have been less investigated than the ones associated with spatial retro-cues.

The present study undertook a systematic investigation of factors that could affect the efficiency of feature-based selection in VWM. Our goals were twofold: (1) To chart the scope of the benefit of attending to features in VWM across several variations in task parameters, and (2) Investigate whether factors specific to feature-, but not spatial-based attention, may disadvantage the feature-based mode of retrieval in VWM, such as the selection of multiple items in the array or the need to present a feature to cue memory that could lead to interference.

In the following sections, we review the current standing in the literature regarding feature-based selection in perception and in VWM and advance four questions related to the robustness of feature-based selection in VWM that we addressed across our studies.

\section{Operation of Spatial and Feature-based Attention in Perception}

Selective spatial attention can optimize visual perception by enhancing the discriminability of stimuli, for example, via increasing contrast sensitivity and spatial resolution (Carrasco, 2011). In contrast, feature-based attention enables the concurrent enhancement of a relevant feature (e.g., color) in multiple locations across the visual field (Carrasco, 2011; Maunsell \& Treue, 2006). Direct comparisons indicate speedier deployment of spatial vs. feature-based attention in perception (300 vs. $500 \mathrm{~ms}$ ), but 
equivalent benefits to performance thereafter (Liu et al., 2007). On the neurophysiological level, spatial attention has been shown to operate via a neural gain mechanism - i.e., by amplifying the neural signal coding information perceived at the attended location - whereas feature-based attention operates by neural tuning, which involves amplification of the relevant signal coupled with suppression of other signals in the visual field (Baldassi \& Verghese, 2005; Ling et al., 2009). In crucial opposition to spatial attention, in which the benefit arises when the selection focus is narrowed, attention to specific features operates in spatially extended manner, i.e., it co-selects goalrelevant features across the whole visual field (Carrasco, 2011; Gledhill et al., 2015; Maunsell \& Treue, 2006; McAdams \& Maunsell, 2000; Saenz et al., 2002; Schledde et al., 2016; Treue \& Trujillo, 1999).

\section{Operation of Spatial and Feature-based Attention in VWM}

Paralleling findings from the perceptual domain, spatial selection in VWM tends to be constrained to one location or to adjacent locations in the memory array, whereas concurrent selection of multiple items via feature-based attention has been observed in VWM (Heuer \& Schubö, 2016; Li \& Saiki, 2015). To date, it is yet not clear to what extant these two types of attention operate by different or similar mechanisms in VWM.

Examinations of feature-based selection have been less extensive and have produced more variable results than spatial selection. For example, some comparisons of the effects of feature-based and spatial retro-cues in VWM have demonstrated that visual features such as color and shape can guide attention as effectively as cues towards spatial locations (Heuer \& Schubö, 2016; Pertzov et al., 2013). In contrast, studies involving two feature-cue dimensions (Gilchrist et al., 2016; Li \& Saiki, 2015) have shown more mixed 
results, indicating either that feature-cues are less beneficial than location cues, or that some feature dimensions may be used more efficiently than others. To explain these findings, Li and Saiki (2015) hypothesized that binding between two features in VWM may be "substantially weaker" than the binding of a feature to a location. One alternative explanation could be that features differ regarding their efficiency for attentional guidance. For example, Heuer et al. (2016) observed smaller benefits when shapes compared to locations were used for cueing. Hence, at present it is not clear whether feature-cueing benefits differ between types of features.

Furthermore, several studies have demonstrated that multi-feature objects demand more VWM capacity (Alvarez \& Cavanagh, 2004; Fougnie et al., 2010; Oberauer \& Eichenberger, 2013), and that memory for visual features is less stable than for spatial locations (Chen \& Wyble, 2015). Thus, the more variable findings with feature retro-cues may result from a trade-off: An increase in the number of representations' features may have diminished the encoding fidelity for them, thus reducing the probability to access one feature via the other, or it may have diminished the ability to create stable bindings between the features to each other and their corresponding location.

In addition, the inconsistent observations of comparable versus differing benefit magnitudes between feature and spatial cues may indicate that feature-location and feature-feature bindings differ in their susceptibility to noise resulting from time-based decay or interference. Studies with spatial retro-cues have ruled out a substantial contribution of protection from time-based decay as an explanation of spatial retro-cue benefits (Gressmann \& Janczyk, 2016; Makovski et al., 2008; Murray et al., 2013; Rerko et al., 2014; Souza, Rerko, \& Oberauer, 2014; Souza, Rerko, Lin, et al., 2014; Souza \& 
Oberauer, 2015; van Moorselaar et al., 2015), but the impact of this factor has not been addressed for feature-based selection. Interference may also modulate the observation of these benefits, a possibility which we will discuss in more detail later.

These mixed findings beg the question whether feature-based selection is robust to various changes in task parameters. Varying these parameters may help to elucidate to what extent feature-based attention operates similarly to spatial attention. Here we considered four questions regarding the mechanisms behind feature retro-cue effects.

\section{Question 1: Is There a Cost to Feature-Based Selection of Multiple Items?}

The study of Heuer and Schubö (2016) revealed that retro-cueing one feature enabled the simultaneous selection of two items in VWM spatially dispersed across the memory array, whereas spatial cueing benefitted two objects only if these were spatially contiguous (see also Makovski \& Jiang, 2007; Matsukura \& Vecera, 2015). However, it is not yet well understood whether this parallel selection comes at a cost in VWM, such as lower precision of the memory representations, or of confusions between the coselected items. A possible scenario when selecting several items in the focus of attention could be that a crosstalk between the attended items emerges, resulting in misattributing one item's feature to the other co-selected item instead, or in an approximation between the items' features, thereby reducing precision of memory.

So far, the majority of the studies using feature retro-cues used recognition tasks (Delvenne et al., 2010; Gilchrist et al., 2016; Heuer \& Schubö, 2016; Li \& Saiki, 2015; Poch et al., 2017). These tasks only permit the examination of how many representations are stored in VWM. Memory quality, however, is also constrained by VWM capacity limitations. The question whether spatial-expanse property of feature-based attention may 
come with some hidden costs or trade-offs requires assessment of memory quality, which is possible with delayed estimation tasks (Prinzmetal et al., 1998; Wilken \& Ma, 2004; Zhang \& Luck, 2008). In these tasks, the visual memoranda vary on continuous dimensions (e.g., colors or orientations). At test, one item's feature is then reproduced from memory on a continuous scale such as a color wheel (Wilken \& Ma, 2004) or by adjusting the orientation with a dial or the mouse (Makovski \& Pertzov, 2015). Memory accuracy is measured by computing the response deviations from the true target feature.

To analyze performance in these tasks, mixture models have been proposed. In the present study, we will focus on the three-parameter mixture model of Bays et al. (2009). This model assumes that responses can come from a (1) distribution reflecting true memory of the tested item, (2) a distribution reflecting true memory for one of the non-tested items which is retrieved instead of the tested item (therefore reflecting misbinding or swap errors), and (3) random guessing. True memory is modeled as a normal distribution centered on the feature value of the tested or one of the non-tested items. The standard deviation of this distribution reflects the precision with which the item is recalled from memory. Random guessing is modeled as a uniform distribution over the feature space. Hence the model has four key parameters: probability of recalling the tested item (PTarget), probability of recalling the non-tested item (PNonTarget), guessing rate (PGuess), and memory precision (Kappa).

Studies with spatial retro-cues have consistently observed increases in the probability of recalling the cued item, and sometimes on the precision of these representations (for a review see Souza \& Oberauer, 2016). To the best of our knowledge, only two studies used delayed estimation task to assess the benefits of feature 
retro-cues through the lens of mixture model parameters. Kalogeropoulou et al. (2017) presented several black and white Gabor gratings across multiple locations. Each color indicated one different orientation. Before the memory array (pre-cue) and during the retention interval (retro-cue), a colored disk indicated the relevant orientation for the test (e.g., black or white) or was neutral (i.e., red). Whereas pre-cues yielded a consistent benefit, retro-cueing did not affect precision, and there was ambiguous evidence for a reduction in guessing. The combined presentation of pre-cues (which were valid on 67\% of the trials) and retro-cues may have diminished participants' motivation to heed the retro-cues, or their ability to do so, if insufficient attention was allocated at encoding to targets that suddenly became relevant with retro-cues.

In the study of Barth and Schneider (2018), participants were presented three colored bars. During the retention interval, a central disk containing one, two, or three colors guided attention to one, two, or all memory items, or a neutral cue was shown (white disk). In half of the trials, retention interference was introduced with presentation of distractor masks. Mixture modelling revealed that only 1-cue trials produced benefits in all mixture model parameters. Cueing multiple items, conversely, produced a cost on memory precision. This result suggests that selection of multiple items may induce precision costs due to cross talk-between multiple attended items. Alternatively, the fact that their 2-cue and 3-cue feature-cue contained multiple colors may indicate that featurecues themselves may introduce interference.

In sum, the scant research with delayed estimation tasks and feature retro-cues does not clearly indicate whether these cues only produce benefits akin to spatial cues or whether they can also incur costs, particularly when multiple items are selected. 


\section{Question 2: Does Feature-Cue Create Interference?}

There is evidence that information presented at test (e.g., the test probe as well as the color wheel) interfere with retrieval, as they introduce visual interference (Souza et al., 2016; Tabi et al., 2019; van Moorselaar et al., 2015). Color wheel interference was observed by Souza et al. (2016): in no-cue trials, participants had a tendency to select a color hue from the wheel section spatially close to the probe. This wheel attraction interference was eliminated both when the wheel was presented in gray color, as well as in a retro-cue condition (Souza et al., 2016). Furthermore, features in change-detection recognition probes have been found to interfere with recall. Retro-cues delay the onset of this perceptual interference as they allow to begin the retrieval ahead of testing (Souza et al., 2016; Tabi et al., 2019).

Retro-cueing features is achieved by either 1) the visual presentation of the cue feature, or 2) with a semantic verbal cue (i.e., 'red', 'circle', as in Gilchrist et al., 2016). When a feature is used as cue, such as a shape (Heuer \& Schubö, 2016; Li \& Saiki, 2015) or orientation (Bays et al., 2011), the cue percept overlaps more strongly with the representation in memory than when the cue is spatial (e.g., an arrow pointing towards a location). It is yet unclear whether presentation of these feature cues create perceptual interference. So far, studies have only compared feature retro-cues to condition with neutral cues which also involves presentation of an irrelevant (uninformative) stimulus during the retention interval. If the neutral cue itself introduces some interference similarly to a visual suffix (Ueno, Allen, et al., 2011; Ueno, Mate, et al., 2011), the estimation of feature retro-cue benefits may depend on the amount of interference created by the neutral cue, and this may explain the variability in benefits observed in the 
literature, and the lack of benefits for 2-cue and 3-cue trials in Barth and Schneider (2018).

\section{Question 3: Does Feature Variability Affect Selection Efficiency?}

Studies employing feature retro-cues have used rather small sets of two to four cue values that always remained constant over trials (Barth \& Schneider, 2018; Heuer \& Schubö, 2016; Kalogeropoulou et al., 2017; Li \& Saiki, 2015). It is not yet known whether feature-based selection in VWM remains as efficient when there are variable cue features to encode from one trial to the next. As feature-encoding has been shown to demand VWM resources (Marshall \& Bays, 2013), higher variability in cue feature values could hamper performance by diverting resources away from the proper encoding of the continuous target feature values (e.g., colors), whereas with often repeating cuefeature values their encoding may be less resource demanding.

\section{Question 4: Is Feature-Based Selection Location-Dependent?}

It has been suggested that feature-based attention in VWM may occur via the mechanism of spatial selection: Namely, the feature retro-cue is used to select the item's location, with location then being used for the retrieval of the to-be-reproduced feature (Pertzov et al., 2013; Pertzov \& Husain, 2014). This conjecture is in line with the idea that the spatial details of the memory array are encoded into VWM and can be retrieved even when not defined as task-relevant (Ester et al., 2018; Günseli et al., 2019; Hajonides et al., 2019; Loaiza \& Souza, 2018, 2019; Myers et al., 2018; Niklaus et al., 2019; Shepherdson et al., 2018; Shepherdson, 2020; Souza et al., 2018)(Chen \& Wyble, 2015; Kondo \& Saiki, 2012; van Ede et al., 2019; Makovski, 2016). As well, there is evidence that focusing on features in VWM recruits brain mechanisms associated with spatial 
attention (Poch et al., 2017), and that binding between two visual features is mediated via spatial location (Bays et al., 2011; Schneegans \& Bays, 2017). Bays et al. (2011) presented colored bars and asked for continuous reports of both feature dimensions. Errors in one feature dimension did not predict errors in the other, indicating that the color and orientation of a given object were not necessarily stored together (see also Schneegans \& Bays, 2017). Pertzov and Husain (2014) presented colored bars either at different locations or at the same location. Same-location trials resulted in significant increase in misbinding errors, namely misattribution of a non-target item's feature to the probed item, compared to when the items were presented in unique locations. However, some studies propose that people can flexibly switch between recourse to either spatialor feature-based strategies in VWM, indicating that the influence of spatial location can be bypassed and feature-feature bindings used directly (Udale et al., 2018).

\section{The Present Study}

Our goal was to systematically probe for the properties of feature-based attention selection in VWM while answering the four questions raised above. To attain this, we conducted six experiments. Table 1 presents a summary of the experiments reported in this paper, the manipulations implemented, and the evidence for feature retro-cue benefits. In Experiment 1, we aimed to replicate feature-based selection when having orientation and color as cued or retrieved visual features. Having established that we could replicate feature-retro-cue benefits with different visual features, in Experiments 2 and 3 we probed whether feature retro-cues could be observed against conditions that control for the contributions of time-based decay and perceptual interference. Next, Experiments 3 and 4 assessed whether feature-cues could benefit recall when two items 
are concurrently selected by the feature-cue. This also allowed us to assess whether cueing two items simultaneously could produce costs to memory either in terms of precision or confusions between cued items. Experiment 4 also allowed us to assess whether variability in cue features values across trials could limit feature retro-cue benefits. Finally, in Experiments 5 and 6 we tested for the role of spatial context in mediating the beneficial effect of feature-based selection.

For all experiments reported here, materials, data, analysis scripts and demonstration videos of the experiments are available on Open Science Framework: https://osf.io/r45a6/?view_only=3f4a5546f72a48d0a6082c9c2d927edb

\section{Experiment 1}

In Experiment 1, we aimed to replicate feature retro-cue benefits using a delayed estimation task, and to assess how this benefit relates to change in different memory parameters as revealed by mixture modelling. We asked participants to memorize colored isosceles triangles that pointed in different orientations. In one block, four discrete colors served as the cue to retrieve continuously varying orientations. In another block, four discrete orientations served as the cue to retrieve continuously varying colors. This allowed us to replicate feature retro-cue benefits with different visual properties. We used fixed cue feature-values to make sure that usage of the retro-cue was not limited by memory imprecision.

To foreshadow our results, we observed that either type of feature cue conferred benefits compared to a neutral-cue condition. This benefit was reflected in an increased probability of retrieving the cued item from memory and on higher memory precision. 


\section{Table 1}

Overview of the Manipulations and Evidence for Feature Retro-Cue Effect in Experiments 1 - 6

\begin{tabular}{|c|c|c|c|c|c|c|c|c|}
\hline \multirow[b]{2}{*}{ Exp. } & \multirow[b]{2}{*}{$\operatorname{Aim}(s)$} & \multicolumn{7}{|c|}{ Manipulations } \\
\hline & & Conditions & $\begin{array}{c}\mathrm{RC} \\
\text { Freq. }\end{array}$ & Cued-Recalled Features & Item:Cue & Trials & $\begin{array}{l}\text { Set- } \\
\text { Size }\end{array}$ & $\begin{array}{l}\text { Evidence (BF) for } \\
\text { Retro-cue benefit }\end{array}$ \\
\hline 1 & $\begin{array}{l}\text { (i) Replicate feature retro-cue effect } \\
\text { with continuous estimation task; } \\
\text { (ii) Establish reliability across different } \\
\text { cued and recalled dimensions. }\end{array}$ & $\begin{array}{l}\text { Cue: Retro- vs. Neutral-Cue } \\
\text { Cue Feature: Color vs. } \\
\text { Orientation }\end{array}$ & $70 \%$ & $\begin{array}{l}\text { (a) Orientation-Color } \\
\text { (b) Color-Orientation }\end{array}$ & $1: 1$ & 396 & 4 & $\begin{array}{l}\text { (a) } 3.1 \times 10^{5} \\
\text { (b) } 1.4 \times 10^{4}\end{array}$ \\
\hline 2 & $\begin{array}{l}\text { Assess cueing effects while controlling } \\
\text { for time-based forgetting and perceptual } \\
\text { interference }\end{array}$ & $\begin{array}{l}\text { (a) Retro-Cue, } \\
\text { (b) No-Cue Short, } \\
\text { (c) No-Cue Long, } \\
\text { (d) Neutral-Cue }\end{array}$ & $50 \%$ & Color-Orientation & $1: 1$ & 396 & 4 & $\begin{array}{l}(\mathbf{a}-\mathbf{b})=12 \\
(\mathbf{a}-\mathbf{c})=92 \\
(\mathbf{a}-\mathbf{d})=224\end{array}$ \\
\hline 3 & $\begin{array}{l}\text { Assess the ability to select two items } \\
\text { concurrently }\end{array}$ & $\begin{array}{l}\text { (a) Retro-Cue, } \\
\text { (b) No-Cue Short, } \\
\text { (c) No-Cue Long, } \\
\text { (d) Neutral-Cue }\end{array}$ & $50 \%$ & Color-Orientation & $2: 1$ & 396 & 4 & $\begin{array}{l}(a-b)=72 \\
(a-c)=1308 \\
(a-d)=1523\end{array}$ \\
\hline 4 & $\begin{array}{l}\text { (i) Replicate selection of multiple items } \\
\text { (ii) Assess impact of variability (high } \\
\text { vs. low) in cue feature values; }\end{array}$ & Cue-set: (a) Small vs. (b) large & $50 \%$ & Shape-Color & $2: 1$ & 400 & 6 & $\begin{array}{l}\text { (a) } 4.76 \times 10^{4} \\
\text { (b) } 7.76 \times 10^{4}\end{array}$ \\
\hline 5 & $\begin{array}{l}\text { Assess whether benefits accrue when all } \\
\text { items share the same spatial location } \\
\text { and whether this depends on post-cue } \\
\text { time }\end{array}$ & $\begin{array}{l}\text { (a) No-Cue, } \\
\text { (b) Retro-Cue } 0.5 \mathrm{~s} \text {, } \\
\text { (c) Retro-Cue } 1 \mathrm{~s} \\
\text { (d) Retro-Cue } 1.5 \mathrm{~s}\end{array}$ & $75 \%$ & Shape-Color & $1: 1$ & 420 & 4 & $\begin{array}{l}(a-b)=2.35 \\
(a-c)=2.37 \\
(\mathbf{a - d})=\mathbf{3 . 2 2}\end{array}$ \\
\hline 6 & $\begin{array}{l}\text { Compare the feature retro-cue effect at a } \\
\text { shared vs. unique spatial locations }\end{array}$ & $\begin{array}{l}\text { Presentation location: (a) } \\
\text { shared-center vs. (b) unique- } \\
\text { periphery }\end{array}$ & $50 \%$ & Shape-Color & $1: 1$ & 208 & 4 & $\begin{array}{l}\text { (a) }=972 \\
\text { (b) }=2.2 \times 10^{5}\end{array}$ \\
\hline
\end{tabular}

Note. Exp. = Experiment, RC Freq. = Retro-cue frequency, BF = Bayes Factor; credible evidence for the benefit of feature-cueing (BF $>3$ ) is marked in boldface. BFs were obtained with $t$-tests. 


\section{Method}

\section{Participants}

Based on previous studies investigating the retro-cue effect in our lab, we planned for sample sizes of at least $N=20$ per experiment. We aimed to report substantial evidence (Bayes Factor, BFs $\geq 3$ or $\leq 0.3$ ) for the main effects and interactions of interest. As Bayesian analyses permit continuous accumulation of evidence (Rouder, 2014), data collection was continued as necessary to arrive at credible evidence for or against an effect, or until we reached a sample size of at least $N=50$.

In Experiment 1, 29 students from Zurich Universities $\left(M_{\text {Age }}=24.5, S D=3.6,7\right.$ men) completed one 90-minute session in exchange for 22.50 Swiss francs or course credit. For all experiments reported here, written informed consent was obtained in accordance with the guidelines of the institutional Ethics Committee, and participants were debriefed at the end of the study.

\section{Procedure}

Experiments 1-5 reported here were programmed in MATLAB (The MathWorks, Natick, MA) using the Psychophysics Toolbox 3 (Brainard, 1997; Pelli, 1997).

The task was to memorize colored isosceles triangles. In one experimental block, color served as the recalled dimension and, in another block, the orientation of the triangle had to be recalled. The feature dimensions of the memoranda were crossed such that one dimension was continuous (i.e., the to-be-recalled one) and the other dimension had four discrete values which served as the retrieval cue. In the color-memory block, four colored isosceles triangles (vertex angle $=30^{\circ}$, height $=100$ pixels) pointing in one of four cardinal orientations $(0,90,180$, and 270) served as the memoranda. The colors 
on each trial were randomly sampled (without replacement) from 360 continuous color values defined in the CIE $L^{*} a * b$ color space $(L=70, a=20, b=38$, and radius $=60)$ that were evenly distributed along a color wheel. In the orientation-memory block, the four colored triangles had orientations randomly sampled from 360 angles, and each triangle was presented in one of four discrete colors (blue, $\mathrm{RGB}=[0,0,255]$; lilac, $\mathrm{RGB}=[138$, $20,236]$; red, $\mathrm{RGB}=[255,0,0]$; and orange, $\mathrm{RGB}=[255,90,0])$.

Task flow is depicted in Figure 1a. In both blocks, the memoranda appeared simultaneously for $1 \mathrm{~s}$, arranged on an invisible circle centered on the middle of the screen (radius $=200$ pixels) against a uniform grey background. This was followed by a blank retention interval of $1 \mathrm{~s}$. On $70 \%$ of the trials, the retention interval ended with presentation of a feature retro-cue in the middle of the screen for $250 \mathrm{~ms}$. In the case of the color-memory block, the feature retro-cue consisted of a white triangle oriented as one of the memory items, which indicated from which item color memory will be probed. In the case of the orientation-memory block, the feature retro-cue consisted of a cross $($ size $=100$ pixels) shown in the color of one of the memory items, which indicated from which item orientation memory will be probed. On the remaining $30 \%$ of the trials, a neutral cue (a white cross, size $=100$ pixels) was drawn in the center of the screen for $250 \mathrm{~ms}$. The offset of the feature retro-cue or the neutral-cue was followed by a $1 \mathrm{~s}$ postcue interval after which the test display was shown. 


\section{Figure 1}

Flow of Events (Panel a), Recall Error (Panel b) and Posterior of the Feature Retro-Cue Effect in each Cue Type Condition (Panel c) in Experiment 1

(a)

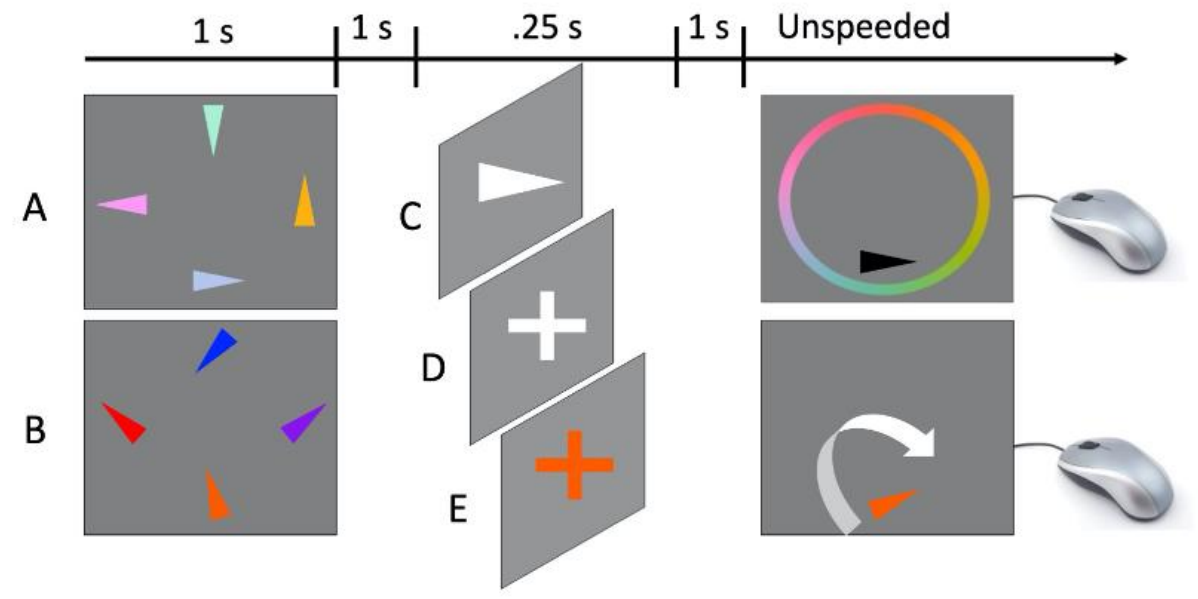

(b)

(c)
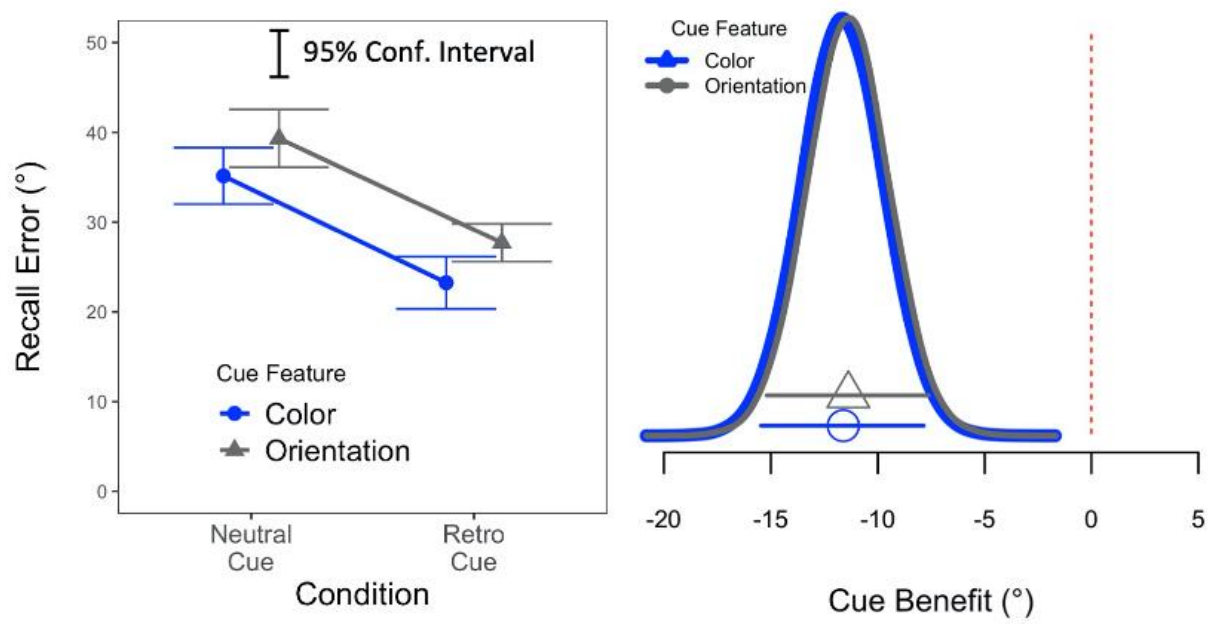

Note. Panel (a) depicts the color (A) and orientation (B) memory arrays, the orientation retro-cue $(\mathrm{C})$, neutral-cue $(\mathrm{D})$ and color retro-cue $(\mathrm{E})$. Panel (b) displays mean recall error per cue type and feature dimension. Panel (c) depicts the posterior distributions of the retro-cue effect (i.e., recall in neutral-cue condition minus the retro-cue condition). Note that only one line is visible, as distributions overlap. 
In the color-memory block, the test display consisted of a color wheel, and a black triangle with the same orientation and in the same location as it had appeared in the memory array (see Figure 1a). Participants had to recall the color of the triangle by selecting a color from the color wheel using the mouse. As participants moved the mouse around the color wheel, the color at the current cursor position was displayed at the probe triangle. Participants confirmed their response by clicking with the mouse button. This was followed by a visual feedback display (duration $=2 \mathrm{~s}$ ) that presented the participant's response and the correct response on the wheel encircled in white and green, respectively.

In the orientation-memory block, the test display consisted of a randomly rotated probe triangle which was drawn in the same color and at the same location as it had appeared in the array (see Figure 1a). Participants used the mouse to adjust the orientation of the probe triangle to the one they remembered. The pointy side of the triangle followed the position of the current mouse cursor. Participants confirmed their response by clicking with the mouse. This was followed by a visual feedback for $2 \mathrm{~s}$ consisting of the probe triangle presented in the position adjusted by the participant, and on top of it, a green triangle frame with the correct orientation.

There were 20 practice trials per block, all with the retro-cue. This was done to assure that participants understood the cueing procedure. Participants were specifically instructed regarding the reliability of the cues and encouraged to use them to guide attention. Each of the two blocks presented 198 test trials, in which retro- and neutral-cue trials were randomly intermixed. Block order was counterbalanced between participants. 


\section{Data Analysis}

The main dependent variable was recall error defined as the absolute distance between the reported feature and the true feature of the tested item. For inference, we conducted Bayesian $t$-tests and analyses of variance (BANOVA), as well as MarkovChain Monte-Carlo (MCMC) sampling of posterior distributions using the BayesFactor package (Morey et al., 2018) in R (R Core Team, 2018). To gauge the effect of cue conditions, we subtracted posterior distributions (retro-cue minus baseline) and evaluated whether the 95\% HDIs (highest density intervals) of the comparison distribution include zero. We regarded Bayes factors (BF) between 3 and 10 as substantial, and larger than 10 as strong evidence for the alternative hypothesis. Conversely, BFs between .30 and .10 were interpreted as substantial, and lower than .10 as strong evidence in favor of the Null hypothesis (Wagenmakers et al., 2011). Ambiguous evidence was indicated by BFs in the range of .3 to 3 .

Mixture modeling was performed with a hierarchical Bayesian measurement model (Oberauer et al., 2017) using JAGS (Plummer, 2016) via the rjags package in R. The hierarchical Bayesian mixture model is an implementation of the mixture model by Bays et al. (2009). The Bayesian estimation method yields probability distributions of parameters' values, providing information about the range of possible parameter values and the uncertainty of the estimates (Oberauer et al., 2017). We fitted the model using 20000 iterations, and discarded 5000 iterations as burn-in. Models' quality checks showed appropriate fit: For all models, the $\hat{R}$ statistic was $<1.02$, indicating that the parallel sampled chains converged to the same posterior distribution. Second, visual inspection of experiment data overlaid with predictions from the models (100 simulated 
subjects per model consisting of a posterior predictive check) showed that the data was captured well by the model (see Supplementary Materials File available on Open Science Framework: https://osf.io/r45a6/?view_only=3f4a5546f72a48d0a6082c9c2d927edb).

\section{Results}

Figure $1 \mathrm{~b}$ shows recall error in each experimental condition. A 2 x 2 repeated measures BANOVA on recall error having cued feature (orientation, color) and cue type (retro-cue, neutral-cue) as predictors showed largest support for a model with only the two main effects $\left(\mathrm{BF}=1.42 \times 10^{10}\right)$. Comparison of the best model against the model including the interaction produced evidence against including the interaction in the model: $\mathrm{BF}_{\text {Interaction }}=3.62 \times 10^{9}$ divided by $\mathrm{BF}_{\text {MainEffects }}=1.46 \times 10^{10}, \mathrm{BF}$ ratio $=0.25$. This provides evidence that cue benefits were of similar magnitude in both feature dimensions. MCMC sampling of posterior distributions revealed credible and similar feature retro-cue benefits for both color and orientation cues (see Figure 1c).

Figure 2 presents the results of the mixture modelling of this data, and Table 2 summarizes mean posterior differences between estimates across cue conditions. Feature retro-cues in both dimensions credibly increased the probability of target recalls (Figure 2a), decreased guessing (Figure 2c), and improved memory precision (Figure 2d)

compared to the neutral cue. In contrast, feature retro-cues incurred no credible change in misbinding errors (probability of recalling a non-target value, see Figure $2 b$ ). 


\section{Figure 2}

Mixture Modeling Parameter Estimates (Mean and 95\% HDI) for Experiment 1

\section{Experiment 1}
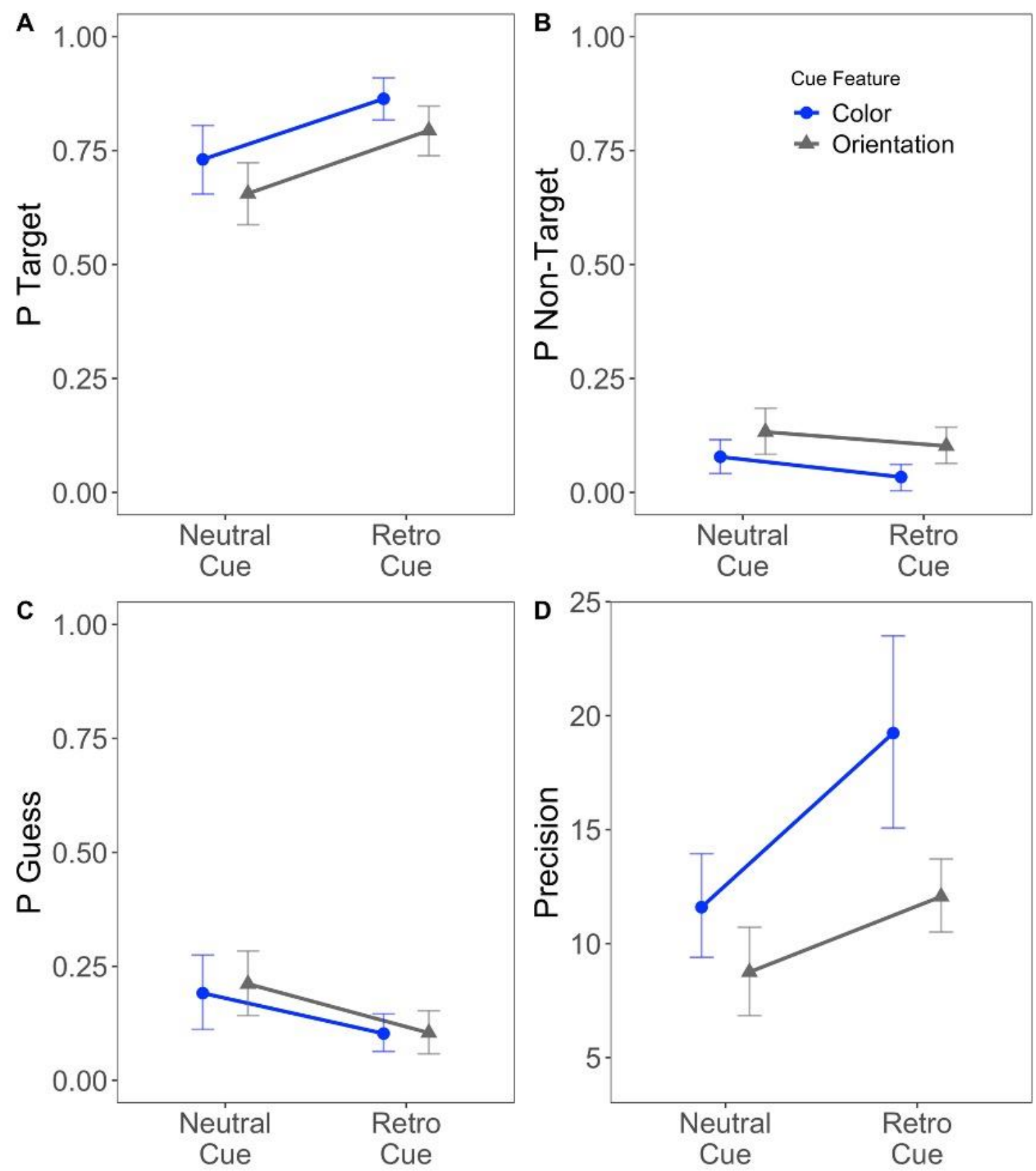
Table 2

Mean and 95\% HDI of the Differences in Posterior Estimates between Cue Conditions in Mixture Model Parameters for all Experiments

\begin{tabular}{|c|c|c|c|c|c|}
\hline \multirow[t]{2}{*}{ Exp. } & \multirow[t]{2}{*}{ Comparison } & \multicolumn{4}{|c|}{ Parameter } \\
\hline & & P Target & P Non-Target & P Guess & Precision \\
\hline \multirow[t]{2}{*}{1} & Orientation-cue $v s$. Neutral & $.138[.051, .226]$ & $-.031[-.097, .034]$ & $-.107[-.192,-.021]$ & $3.314[.806,5.861]$ \\
\hline & Color-cue vs. Neutral & $.133[.044, .221]$ & $-.044[-.092, .003]$ & $-.089[-.182,-.001]$ & $7.641[2.875,12.513]$ \\
\hline \multirow[t]{3}{*}{2} & Retro-cue vs. Short & $.046[-.029,0.122]$ & $-.054[-.108, .001]$ & $.008[-.061, .075]$ & $4.699[.778,8.739]$ \\
\hline & Retro-cue vs. Long & $.092[.008, .178]$ & $-.043[-.087, .001]$ & $-.049[-.136, .035]$ & $4.111[.647,7.804]$ \\
\hline & Retro-cue vs. Neutral & $.056[-.015, .126]$ & $-.023[-.071, .024]$ & $-.033[-.104, .034]$ & $5.167[.904,9.305]$ \\
\hline \multirow[t]{3}{*}{3} & Retro-cue vs. Short & $.041[-.025, .106]$ & $-.011[-.058, .032]$ & $-.031[-.091, .031]$ & $-.294[-4.515,3.812]$ \\
\hline & Retro-cue vs. Long & $.058[-.008, .126]$ & $-.036[-.086, .016]$ & $-.021[-.081, .034]$ & $1.583[-1.996,5.031]$ \\
\hline & Retro-cue vs. Neutral & $.063[.004, .123]$ & $-.033[-.079, .011]$ & $-.031[-.082, .023]$ & $2.503[-.945,5.932]$ \\
\hline \multirow[t]{4}{*}{$2 \& 3$} & Short 1 vs. Short $2 *$ & $-.029[-.107, .051]$ & $-.011[-.048, .072]$ & $.018[-.054, .091]$ & $-3.422[-7.676, .482]$ \\
\hline & Long 1 vs. Long $2 *$ & $-.058[-.147, .029]$ & $-.026[-.084, .031]$ & $.084[-.001, .172]$ & $-.988[-3.995,1.859]$ \\
\hline & Neutral 1 vs. Neutral $2 *$ & $-.017[-.088, .053]$ & $-.043[-.096, .011]$ & $.061[-.007, .126]$ & $-1.073[-4.738,2.637]$ \\
\hline & Retro 1 vs. Retro $2 *$ & $-.043[-.105,-.019]$ & $-.017[-.054, .021]$ & $.061[.005, .116]$ & $1.174[-2.915,5.354]$ \\
\hline \multirow[t]{4}{*}{4} & Retro 6 - Neutral 6 & $.114[.046, .182]$ & $.014[-.055, .085]$ & $-.127[-.199,-.059]$ & $1.307[-.141,2.851]$ \\
\hline & Retro 3 - Neutral 3 & $.081[.011, .149]$ & $-.044[-.116, .029]$ & $-.035[-.102, .032]$ & $1.183[-.623,2.981]$ \\
\hline & Retro 6 - Retro 3 & $.024[-.048, .097]$ & $.018[-.057, .091]$ & $-.042[-.101, .017]$ & $-1.024[-2.882, .778]$ \\
\hline & Neutral 6 - Neutral 3 & $-.011[-.075, .055]$ & $-.041[-.109, .028]$ & $.051[-.027, .125]$ & $-1.148[-2.685, .293]$ \\
\hline \multirow[t]{3}{*}{5} & 0.5 sec. Post-Cue - No-Cue & $.035[-.009, .079]$ & $-.014[.059, .032]$ & $-.021[-.072, .029]$ & $1.685[-.322,3.738]$ \\
\hline & 1 sec. Post-Cue - No-Cue & $.026[-.018, .069]$ & $-.022[-.067, .022]$ & $-.004,[-.058, .048]$ & $1.771[-.177,3.703]$ \\
\hline & 1.5 sec. Post-Cue - No-Cue & $.047[.001, .093]$ & $-.019[-.066, .028]$ & $-.028[-.078, .021]$ & $1.018[-1.071,3.241]$ \\
\hline \multirow[t]{2}{*}{6} & Center (shared location) & $0.036[0.002,0.071]$ & $-0.022[-0.057,0.014]$ & $-0.015[-0.063,0.033]$ & $0.334[-0.564,1.203]$ \\
\hline & Periphery (unique locations) & $0.067[0.027,0.107]$ & $-0.068[-0.105,-0.031]$ & $0.001[-0.054,0.053]$ & $0.811[-0.062,1.702]$ \\
\hline
\end{tabular}

Note. The point estimates are means and 95\% Highest Density Intervals (in square brackets). Credible effects (i.e., HDIs that do not include zero) are in boldface. * In the joint modeling of Experiments 2 and 3, the numbers in the Comparison column denote the number of items that shared the cue feature. 


\section{Discussion}

Experiment 1 showed robust benefits both when orientation and color were used as cues to allow for retrieval of the other feature from VWM. Mixture modeling revealed that feature retro-cues considerably increased the probability of retrieving the tested information and reduced guessing, showing that these cues increased the accessibility of the cued information. These results parallel the ones obtained for spatial retro-cues (see Souza \& Oberauer, 2016). Feature retro-cues were also associated with heightened memory precision in Experiment 1. Benefits for memory precision have been sometimes reported for spatial retro-cues as well, but not very consistently. Usually, studies that observed spatial retro-cue benefits on precision have used small set-sizes (less than four items), whereas for higher set-sizes benefits accrued only for recall probability (see Souza \& Oberauer, 2016, for a review). Experiment 1 also used a relatively small setsize. Across all experiments reported here, however, benefits on memory precision were not consistent, indicating that this effect is not robust for feature retro-cues as well.

\section{Experiment 2}

Experiment 1 established that feature retro-cues improve performance in a delayed estimation task. Experiment 2 builds on this finding and extends it by examining two hypotheses regarding the mechanisms behind the retro-cue effect, the first concerning protection from time-based forgetting, and the second regarding protection from perceptual interference.

First, it has been demonstrated that spatial retro-cues benefit memory in more extensive ways than simple protection from time-based forgetting (Makovski et al., 2008; Murray et al., 2013; Rerko et al., 2014; Souza, Rerko, Lin, et al., 2014; Souza \& 
Oberauer, 2015). Given that feature-feature bindings have been suggested to be less robust than feature-location bindings (Bays et al., 2011; Li \& Saiki, 2015), it is unclear whether these bindings are more susceptible to time-based forgetting, and if feature-cue benefit protects representations from it. Secondly, it is known that visual stimulation can have a disrupting effect on VWM performance, which is the case with visual information displayed at test (Souza et al., 2016; Tabi et al., 2019). Concerningly, feature retro-cues present information that is similar to the memoranda, which may result in interference at the cueing time-point, thus limiting the scope of feature retro-cue benefits. This would be the case if feature retro-cues were only beneficial compared to a neutral-cue condition, which consists of presentation of a non-informative visual event during the retention interval that may nevertheless interfere with VWM akin to a visual suffix (Ueno, Allen, et al., 2011; Ueno, Mate, et al., 2011). Neutral-cues have been shown to induce lower performance than no-cue conditions in an orientation task (Murray et al., 2013). If neutral-cues induce interference, they may harm performance more than the feature retrocue, yielding then a feature retro-cue benefit.

We addressed these two issues by introducing different baseline conditions to compare the feature retro-cue condition to. Firstly, if feature-feature bindings suffered from time-based forgetting and feature-cues only buffered against this disadvantage, there should be a benefit when the retro-cue condition is compared against a long no-cue baseline, which matches the total retention interval in the retro-cue condition, but not against a short no-cue baseline, whose retention ends at the same time as the cue is presented in retro-cue trials. Secondly, if feature retro-cues themselves incurred perceptual interference, then their benefit should emerge compared to the neutral-cue 
condition which is matched for perceptual stimulation, but not against baselines

presenting a blank screen (no-cue long and short).

We chose orientation as the to-be reported feature in Experiments 2 and 3 because Experiment 1 showed commensurate recall accuracy, as well as commensurate retrocueing benefits, across the color and orientation feature dimensions. In Experiments 4, 5, and 6 we then used color as the recalled feature.

\section{Method}

Twenty new university students from the Zurich area $\left(M_{\text {Age }}=24.2, S D=3.84,5\right.$ men) completed one 90-minute session in exchange for 22.50 Swiss francs or course credit. As in Experiment 1, the task was to remember the orientations (ranging from 1$360^{\circ}$ ) of four isosceles triangles, each presented in one of four discrete colors (blue, RGB [0 0 255]; violet, RGB [138 20 236]; red, RGB [255 0 0]; and orange, RGB [255 90 0]; see Figure 3a). The memoranda were shown for $1 \mathrm{~s}$, followed by a $1 \mathrm{~s}$ retention interval. On $50 \%$ of the trials, the retention interval was followed by the presentation of a color retro-cue (i.e., cross, size $=100$ pixels, duration $=250 \mathrm{~ms}$ ) displayed in the middle of the screen. The retro-cue was shown in the color of the to-be-tested item and indicated it with $100 \%$ validity. The offset of the retro-cue was followed by a 1 -s post-cue interval. The remaining $50 \%$ of the trials were divided equally between three baselines: Short no-cue, long no-cue, and neutral cue. To test the time-based forgetting hypothesis, the short nocue baseline consisted of presentation of the test display at the time point when the retrocue would have occurred. In contrast, the long blank baseline was matched in duration to the total time until test in the retro-cue condition. To test the interference hypothesis, neutral-cue trials presented a white cross (size $=100$ pixels) instead of the retro-cue. This 
matches the retro-cue condition in terms of timing and controls for perceptual

stimulation, but conveys no information to guide attention.

Figure 3

Illustration of the Flow of Events (Panel a), Mean Recall Error Across Conditions (Panel b), and Posterior of the Feature Retro-Cue Effect Computed Against the Different Baselines (Panel c) in Experiment 2

(a)

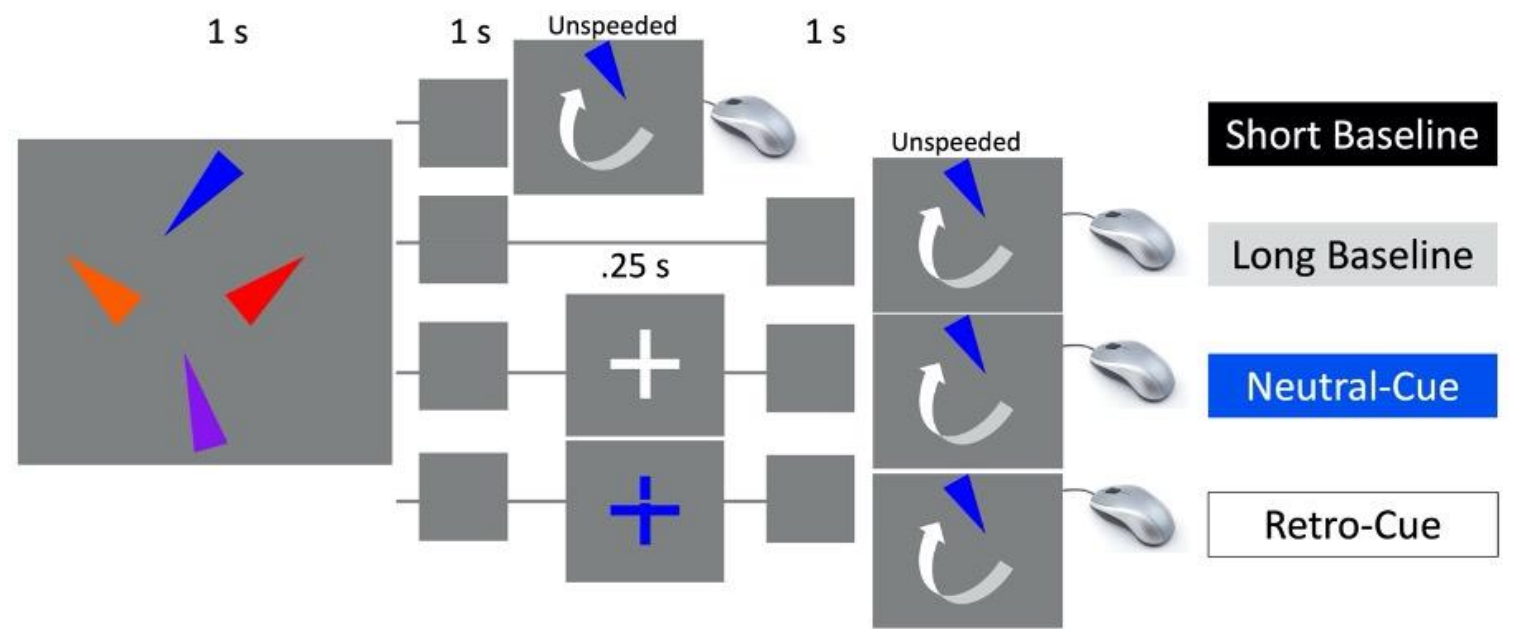

(b)

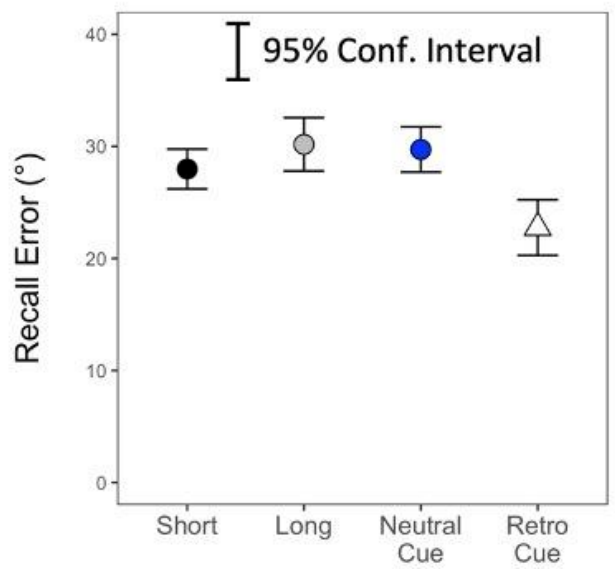

(c)

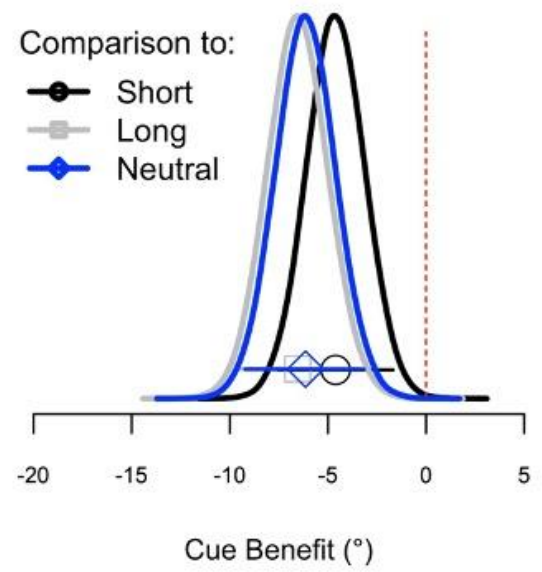


At test, a randomly rotated probe triangle was shown in one of the locations occupied by the memory items, in its original color. Participants rotated the tip of the probe triangle to match the item's original orientation from memory, and they confirmed their response with a mouse click. Visual feedback was provided as in Experiment 1. There were 20 practice trials, all with retro-cues, followed by 396 experimental trials in which all experimental conditions were randomly intermixed.

\section{Results}

Figure $3 b$ presents mean recall error in Experiment 2. A one-way BANOVA having cue condition (retro-, neutral-, short no-cue, long no-cue) as a predictor yielded overwhelming evidence for the effect of condition $(\mathrm{BF}=1520)$. Bayesian $t$-tests demonstrated credible differences between the retro-cue condition and each baseline (BF short no-cue $=12$, long no-cue $=92$, neutral-cue $=224$ ), but not between the baselines themselves $(\mathrm{BF}$ short no-cue vs. long no-cue $=0.80 ;$ short no-cue vs. neutral-cue $=0.60$; long no-cue vs. neutral-cue $=0.24)$. Comparison of MCMC-sampled posterior distributions showed credible retro-cue benefits against every baseline (see Figure 3c), with baselines not differing credibly from each other.

Results of the mixture modeling are shown in Figure 4 and credibility of differences between conditions is summarized in Table 2. The retro-cue tended to increase the probability of recalling the target, but this effect was only credible when the retro-cue was contrasted against the long no-cue baseline. Feature retro-cues also somewhat decreased the probability of non-target recalls, but again this effect was not fully credible. Guessing was not credibly modulated by condition. With regard to 
memory precision, however, there was a credible increase in the retro-cue condition compared to all baselines.

\section{Figure 4}

Mixture Modeling Parameter Estimates (Mean and 95\% HDI) for Experiment 2

\section{Experiment 2}
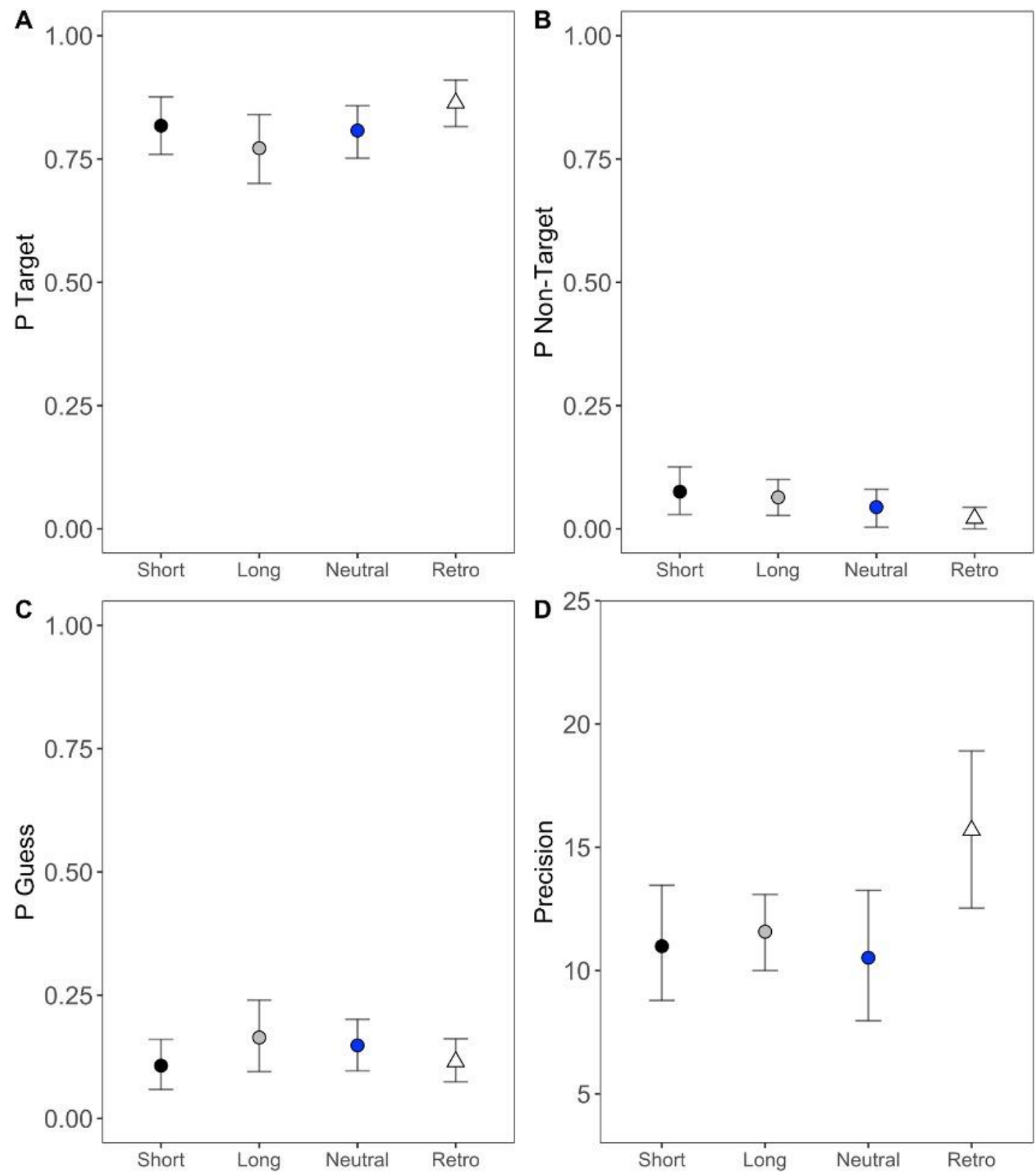


\section{Discussion}

Experiment 2 evaluated two hypotheses pertaining the mechanisms behind feature retro-cue benefits: Protection from (a) time-based forgetting, and (b) visual interference by the cue.

To assess protection from time-based forgetting, we implemented a short baseline (matched to the time prior to the onset of the retro-cue, namely $1 \mathrm{~s}$ ), and a long baseline (matching the retro-cue trial duration, namely $2.25 \mathrm{~s}$ ). Critically, these two baseline conditions did not credibly differ from each other, and feature retro-cue benefits were observed against both. This indicates that feature retro-cues are not buffering against rapid degradation of feature-feature bindings during short retention intervals up to $2 \mathrm{~s}$. Note that performance may deteriorate when longer maintenance durations are used (see Pertzov et al., 2013; Zhang \& Luck, 2009), and protection from this time-based forgetting may start to contribute to feature retro-cue benefits.

To assess perceptual interference by feature cues, we included a neutral-cue condition that involves presenting a visual stimulus at the same time as the feature retrocue would appear, and a long no-cue condition that did not involve any visual stimulation during the retention interval. We reasoned that a sudden onset stimulus (neutral-cue or feature retro-cue) could interfere with memory, disrupting it akin to a visual suffix effect (Ueno, Allen, et al., 2011; Ueno, Mate, et al., 2011). However, neutral-cue trials yielded similar performance to long no-cue trials, with performance in retro-cue trials being the best. This indicates that the sudden onset of the neutral- and retro-cue did not disrupt memory maintenance in our experiment. This is inconsistent with the hypothesis that 
feature retro-cues would only benefit memory in comparison to conditions that also involve some degree of perceptual interference.

Mixture modelling showed benefits for memory precision, but not credibly on probability of recall. This contrasts with the observation in Experiment 1, in which both accessibility and precision were improved by retro-cues. One possible explanation why the two experiments differ in terms of improvements in model parameters may relate to the frequency of the retro-cue trials: In Experiment 1, 70\% of the trials involved retrocues, whereas in Experiment 2, this was reduced to $50 \%$ to accommodate the presentation of the other baselines. More frequent exposure to the retro-cue might be required for larger benefits on memory parameters to amass.

\section{Experiment 3}

Experiment 3 was designed to, on the one hand, replicate the important facility of feature attention in the perceptual domain - namely, selection of multiple items across the visual field, - and on the other hand, to explore with mixture modelling how memory is influenced when attention prioritizes two instead of one item in VWM. The main aim was to test whether attentional selection of two representations in VWM diminishes precision (indicating an approximation of the co-selected feature values), incurs larger binding errors, or both. To this end, we presented arrays in which two elements were mapped to a single cue (i.e., a shared color). Accordingly, when the feature retro-cue was presented, it indicated two items as candidates to be tested, each with $50 \%$ chance (see Figure 5a for the depiction of the task flow). This allowed us to examine if the cue permits the simultaneous selection of these two items. Then, we applied mixture modeling to joined data of Experiments 2 and 3 to evaluate changes associated with 
increase in the number of cued representations. If feature retro-cues can select multiple items across the memory array, then feature retro-cue benefits in Experiment 3 should be as reliable and as large as in Experiment 2.

\section{Method}

Experiment 3 included a new sample of 21 university students from the Zurich area $\left(M_{\text {Age }}=23.7, S D=2.7,7\right.$ men $)$ that completed one 90 -minute session. As in Experiment 2, participants memorized and reproduced the orientations of four randomly rotated triangles. All conditions and materials were identical to Experiment 2, except that on every trial two items shared the same color. The colors were randomly sampled from the four colors used in Experiment 2. Accordingly, the color retro-cue indicated two items simultaneously as candidates for being tested at the end. Each of the cued items had $50 \%$ chance of being tested. At test, the probe appeared in its original position in the memory array, and participants adjusted its orientation to the one they remembered.

\section{Results}

Figure $5 \mathrm{~b}$ presents recall error across the four conditions. A one-way BANOVA with the factor cue condition (retro-cue, neutral-cue, short no-cue, and long no-cue) yielded strong support for the effect of condition $(\mathrm{BF}=6980)$. As illustrated in Figure $5 \mathrm{~b}$, this is mainly due to lower recall error in the retro-cue condition compared to the remaining conditions. Bayesian $t$-tests showed credibly different performance in the cue condition compared to every baseline $(\mathrm{BF}$ short no-cue $=72$; long no-cue $=1308$, neutral-cue $=1523)$, whereas the baselines did not credibly differ $($ BF short-long $=0.59$; short-neutral $=0.57$; long-neutral $=0.22$ ). Similar to Experiment 2, the MCMC sampling 
of model's posterior distributions showed credible decrease in recall error in the retro-cue condition against every baseline (see Figure 5c).

\section{Figure 5}

Illustration of the Flow of Events (Panel a), Mean Recall Error Across Conditions (Panel b), and Posterior of the Feature Retro-Cue Effect Computed Against the Different Baselines (Panel c) in Experiment 3

(a)

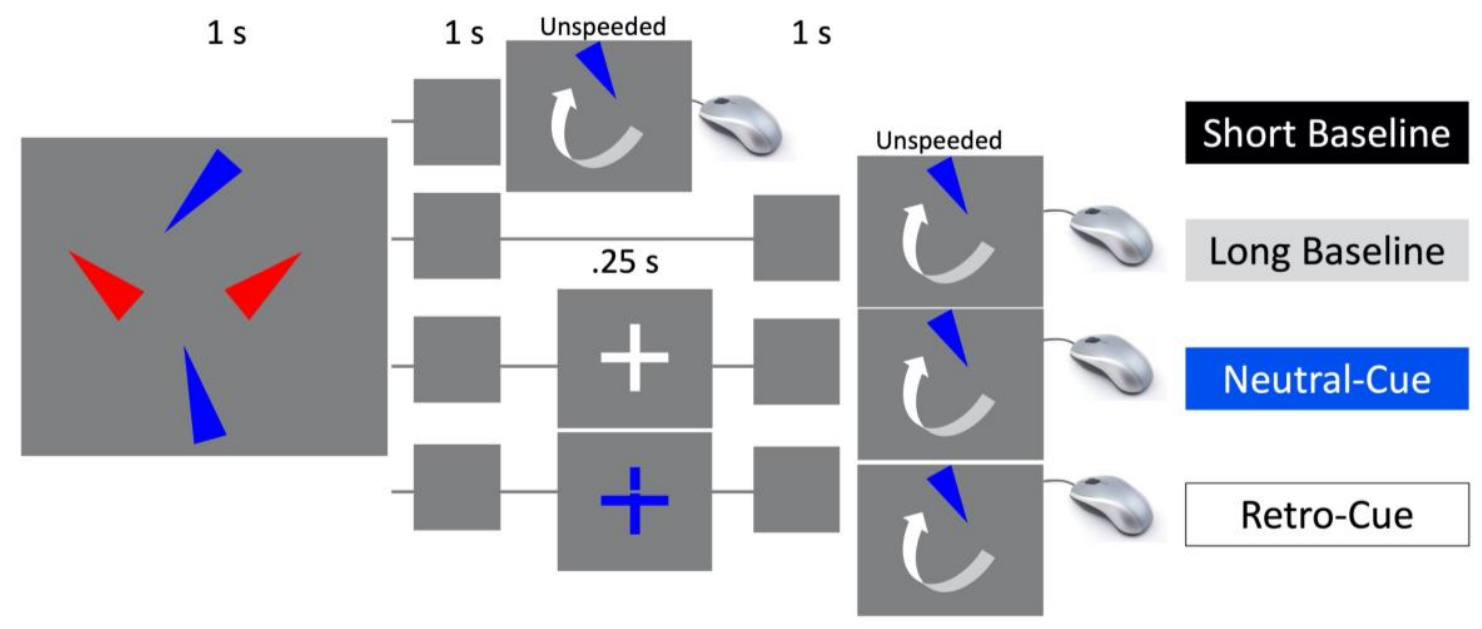

(b)

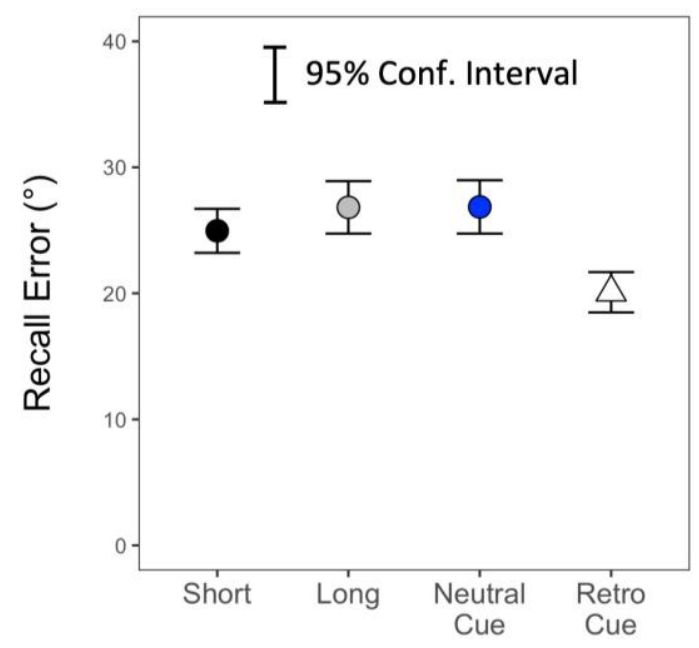

(c)

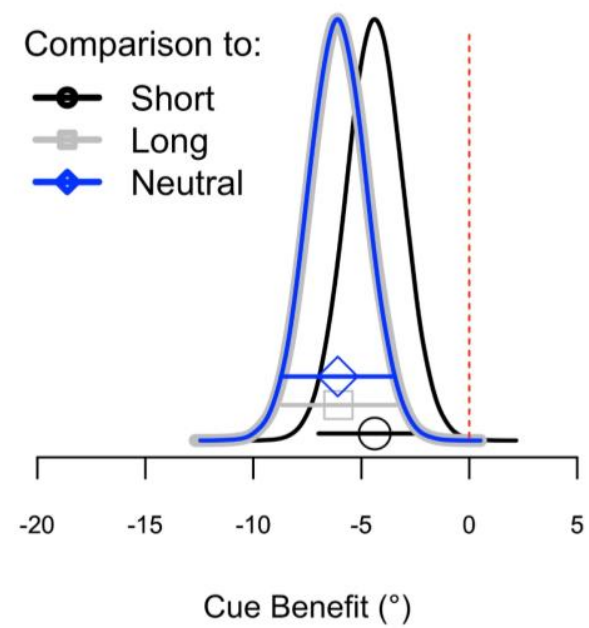


Given that the retro-cue tagged two out of the four array items, we additionally examined whether recall error differed when two adjacent $(M=19.62, S D=29.31)$ compared to two diagonally positioned items $(M=20.98, S D=33.57)$ were retro-cued. A Bayesian $t$-test did not show evidence for a difference, $\mathrm{BF}=0.55$.

Mixture model of the data of Experiment 3 (see Figure 6 and Table 2) showed higher probability of recalling the target in the retro-cue condition, but this increase was only fully credible compared to the neutral baseline. There was a tendency for lower probability of recalling non-target items and a reduction in guessing, but these changes were again not fully credible. Importantly, these results go against the notion that cueing two instead of one item for retrieval in VWM may increase the probability of swap errors, which predicts higher rates of NonTarget responses. Indeed, probability of recalling another item (PNonTarget) was generally low $(<.10)$, and, crucially, these values were lowest in the Retro-cue condition (Mean [95\% HDI]: Retro-cue $=.05[.02$, $.07]$, short baseline $=.06[.02, .102]$, long baseline $=.08[.04, .13]$, Neutral-cue $=.08[.04$, .12]). Regarding memory precision, there was also no credible evidence for a change in precision in the retro-cue condition, which argues against the concern that double-item cueing may lead to approximation of representations of the two cued items in VWM. These results suggest that the overall retro-cue benefit observed in the recall error measure was spread across several parameters such that it could not be credibly ascribed to a particular source, but that double-cueing did not lead to disadvantages. This was further confirmed by the joint modelling of Experiment 2 and 3, reported next. 


\section{Figure 6}

Mixture Modeling Parameter Estimates (Mean and 95\% HDI) for Experiment 3

\section{Experiment 3}
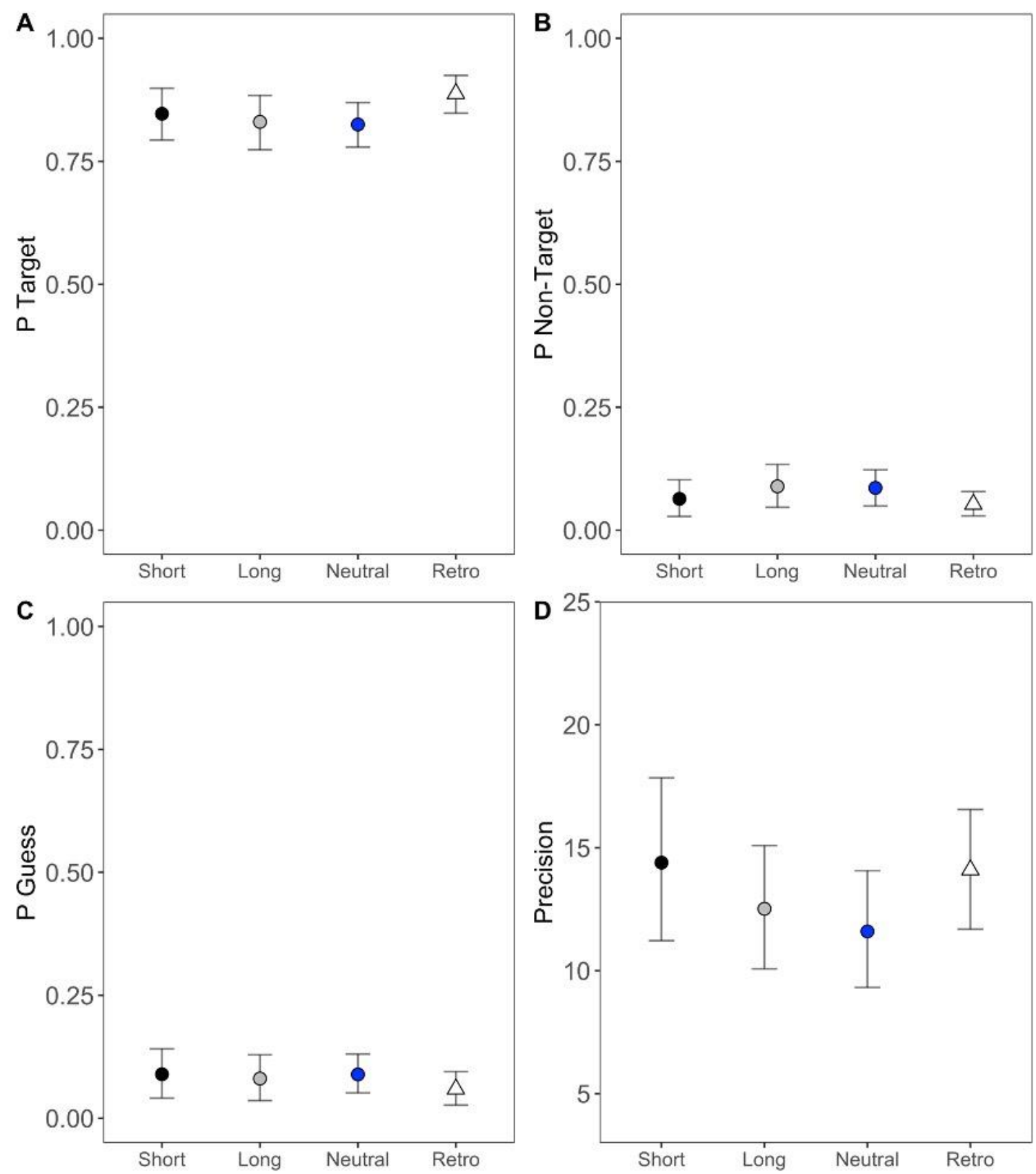


\section{Discussion}

In Experiment 3, the recall error pattern was similar to Experiment 2 and the analyses showed strong support for retro-cue effect against all baselines. However, mixture modelling did not provide credible indication for advantages in one particular memory parameter as a function of retro-cueing. One may wonder if in the light of strong similarity in performance pattern between Experiment 2 and 3, the absence of improvement in the precision parameter may indicate hidden cost of directing attention to two memory representations. We addressed this question with a combined mixture modelling of both experiments' data.

\section{Combined Analysis: Experiments 2 and 3}

To investigate how attentional selection of one versus two items in VWM changes the feature retro-cue effect, we modelled the data of Experiments 2 and 3 jointly. The mixture modelling parameters are visualized in Figure 7 and differences between posteriors across equivalent conditions are provided in Table 2.

Comparisons of both experiments' baselines revealed no credible differences. Comparisons of cued conditions showed that performance in the two-item cue condition (Experiment 3) was not inferior compared to when only one item was cued (Experiment 2). Unexpectedly, one credible difference emerged in the opposite direction: Probability of guessing was lower when two items were cued compared to one. 


\section{Figure 7}

Raw Recall Error in Experiment 2 cueing one item (Panel a) and Experiment 3 cueing two items (Panel b), and Parameter Estimates (Mean and 95\% HDI) for the Joint Modeling of the Data of Experiments 2 and 3 (Panel c)

(a)

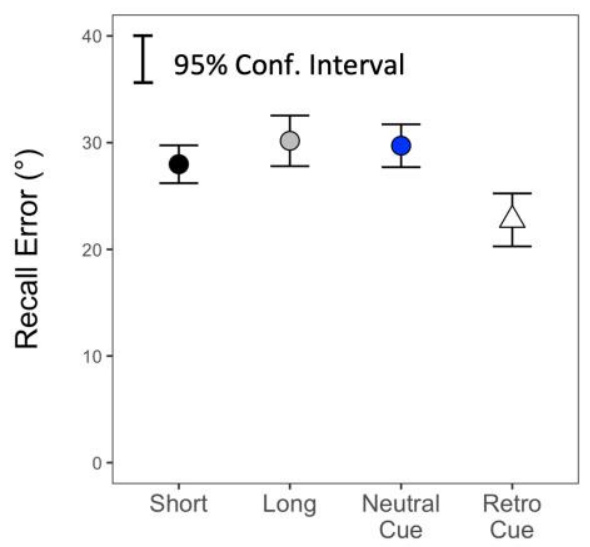

(b)

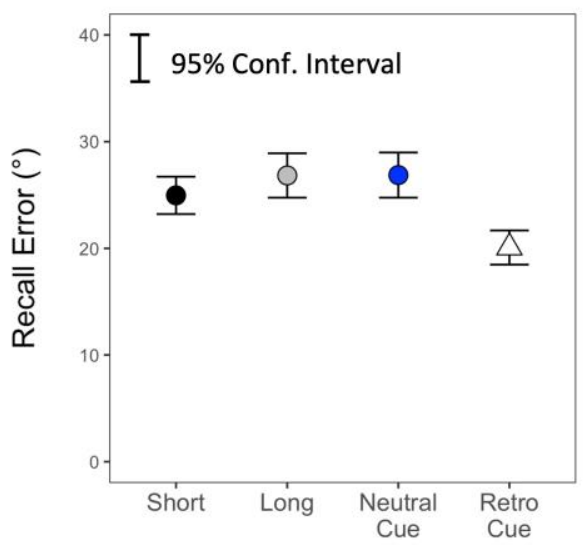

(c)

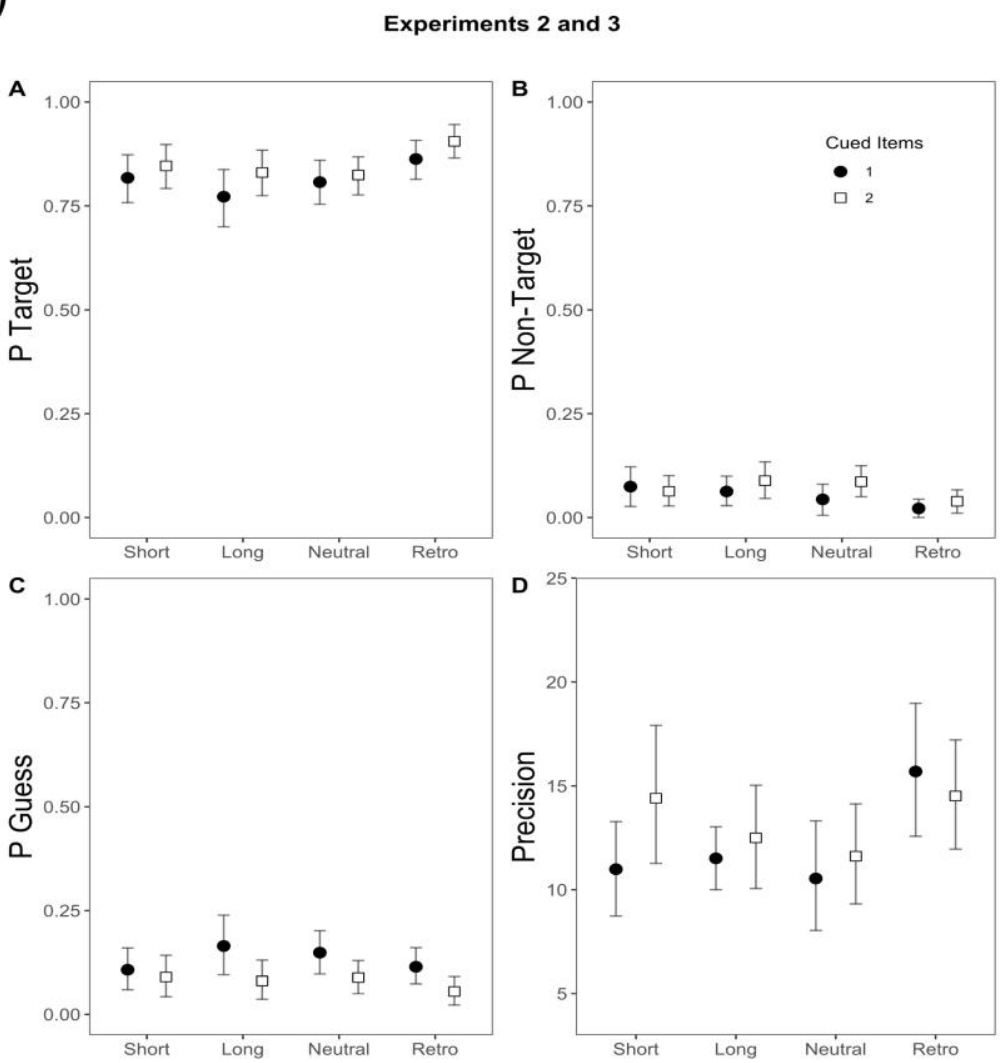


Together, these results argue against the two main concerns pertaining to doublecueing of representations in VWM: Firstly, there was no increase in swap errors, indicating that cued items did not interfere with each other at recall. Secondly, no credible differences in memory precision emerged, arguing against the notion that recalling two items in VWM may result in approximation of the co-selected items' feature values.

\section{Experiment 4}

One aim of Experiment 4 was to replicate one important feature of Experiment 3, namely the concurrent selection of multiple items via feature-based attention. This allowed us to further establish whether parallel selection would be associated with only memory benefits or whether costs could be observed in some memory parameters. We did so while changing the type of recalled feature (color instead of orientation), cue-type (shapes instead of colors), and set-size (6 instead of 4). Experiment 4 therefore generalizes the results of Experiment 3.

In addition, we used Experiment 4 to explore one further issue that may affect the robustness of feature-based selection. The capacity of VWM is limited by both the number of objects stored and the number of features within the object (Fougnie et al., 2010; Hardman \& Cowan, 2015; Oberauer \& Eichenberger, 2013). Feature retro-cue tasks involve the encoding of multi-feature items, hence performance in this task is limited by the ability to bind the cue and the target features, together with their location. Arguably, binding of features requires more cognitive resources as memory for locations, the latter being encoded even regardless of its task-relevance (Chen \& Wyble, 2015; Kondo \& Saiki, 2012; van Ede et al., 2019). For example, Chen and Wyble (2015) found 
that pre-cues' locations could be recalled regardless whether these cues were relevant to the task, whereas recall for the pre-cues' features was poor even when these were paramount for the successful task completion. In other words, whereas memory for stimulus locations in VWM was unintentional and independent of their task relevance, recall of features was less robust (Chen \& Wyble, 2015).

Recurring cue-features (e.g., shapes) may leave more available resources for encoding, thereby enabling higher memory precision. Previous work with feature retrocues used small sets of recurring feature-cue values, i.e., three (Heuer \& Schubö, 2016) and four (Li \& Saiki, 2015). Here, we asked whether feature-cues can be less efficiently utilized when contrasting lower and higher across-trial variability of the cue-feature set (i.e., three vs. six). The critical manipulation consisted in blocked presentation of fixed sets of three shapes (which always reoccurred in every trial) or random sampling of three shapes out of a pool of six (which incurred trial-to-trial variability in the shapes) to cue colors in memory. Thus, the critical difference between conditions consisted in the rate at which shape values changed between trials. Observing reduced efficiency with higher cue value turnover would demonstrate that encoding of variable feature values across trials consumes more VWM capacity.

\section{Method}

A new sample of 56 university students $\left(M_{\text {Age }}=24.7, S D=3.69,14\right.$ men $)$ completed one session in Experiment 4. We manipulated two factors: Number of shapes used as cue (3 vs. 6, within-subjects), and shape complexity (simple vs. complex shapes; between-subjects). The latter served only as a control factor, since we were not interested in shape complexity. We planned to collect a sample of $\mathrm{N}=28$; however, after this 
sample size was reached there was ambiguous evidence regarding whether shape complexity had an effect. Hence, we decided to double the sample size to assess whether effects of shape complexity would become credible, which was not the case.

Figure 8a presents the visualizations of the experimental stimuli and the task flow. Participants remembered six colored shapes (radius $=35$ pixels). The shapes were presented simultaneously, arranged on an invisible circle (radius $=200$ pixels) centered in the middle of the screen. Per array, two items always shared one shape. Selection of the shapes in each trial varied depending on the block. In the small cue-set block, shapes were selected from a fixed pool of three shapes: Either from the set of: circle, square and triangle, or from the set of half-moon, cross, and star. Hence, the same shapes reoccurred on every trial, associated with different colors. In the large cue-set block, three shapes were randomly selected from a pool of six (i.e., circle, square, triangle, half-moon, cross, and star). Accordingly, in the large cue-set block, there was variability in which shapes were shown on each trial. Half of the participants were assigned to the small cue-set with the triangle, square, and circle shapes, and the other half to the small cue-set with the half-moon, star, and cross shapes. This allowed us to assess whether there was any impact of having very basic (i.e., triangle) or putatively more complex (i.e., star) shapes as cues per se, which may contribute to cue-use in the large cue-set block.

For every trial, colors were randomly sampled from the same color wheel as used in Experiment 1. The trial proceeded as follows: The memory array was presented onscreen for $1 \mathrm{~s}$, followed by a 1-s blank retention interval, the presentation of a retro-cue or neutral-cue for $250 \mathrm{~ms}$, and a subsequent 1-s blank post-cue interval which ended with the test display. The retro-cue ( $50 \%$ of the trials) consisted of a white shape (radius $=35$ 
pixels) that indicated that only the two memory items associated with this shape were relevant for the upcoming test, with any of these items being equally likely to be tested. The neutral-cue consisted of a thin white cross ( $\operatorname{size}=100$ pixels). At test, a probe shape was drawn in dark grey $(\operatorname{RGB}=[90,90,90])$ at its original location in the memory array. The shape always matched the shape of the item presented at that location. The probe shape was presented together with the color wheel and the mouse cursor. Participants selected the color of the item from the color wheel and confirmed their response with a mouse click. This was followed by presentation of visual feedback. There were 20 practice trials, all with retro-cues, and 200 test trials per block. Cue conditions were randomly intermixed in each block. Block order was counterbalanced across participants.

\section{Results}

\section{Control Analyses}

First, we assessed the effect of the shape-complexity factor that was manipulated between-subjects in the small cue-set block. We compared recall error between the two groups of participants, having cue condition as an additional predictor. The best model of the data included only the main effect of cue condition $\left(B F=3.46 \times 10^{4}\right)$. There was ambiguous evidence against a main effect of shape complexity $(\mathrm{BF}=0.57)$. Furthermore, there was also ambiguous evidence against including the interaction between shape complexity and cueing $\left(\mathrm{BF}_{\text {Interaction }}=7933\right.$ divided by $\mathrm{BF}_{\text {MainEffects }}=19970, \mathrm{BF}-$ ratio $=$ 0.39). We also conducted $t$-tests between the shape complexity groups for the neutraland retro-cued conditions separately. Recall error did not differ between simple $(M=56$, $S D=13)$ and complex shapes $(M=58, S D=14)$ in the neutral-cue condition $(\mathrm{BF}=0.3)$. Simple $(M=48, S D=15)$ and complex shapes $(M=52, S D=12)$ also tended not to 
differ in the retro-cued condition $(\mathrm{BF}=0.49)$. Together, we took this evidence to indicate that the types of shapes used did not substantially influence performance, and henceforth treated them as one factor level (small cue-set).

\section{Figure 8}

Illustration of the Stimuli and the Flow of events in the Task (Panel a), Recall Error Across Conditions (Panel b) and the Posterior of the Retro-Cue Effect (Panel c) in Experiment 4

(a)

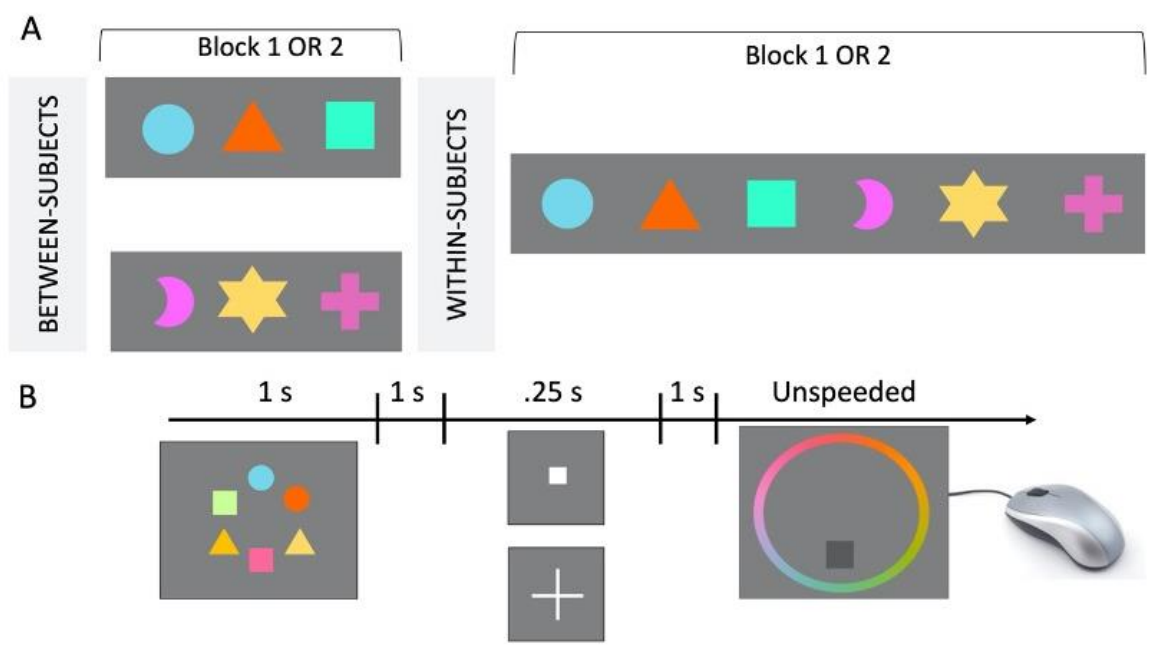

(b)

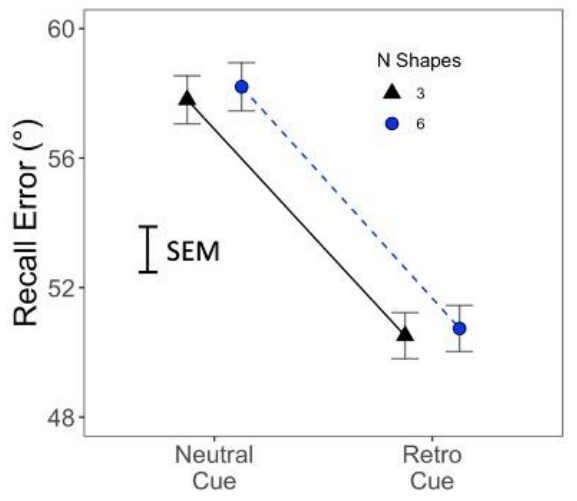

(c)

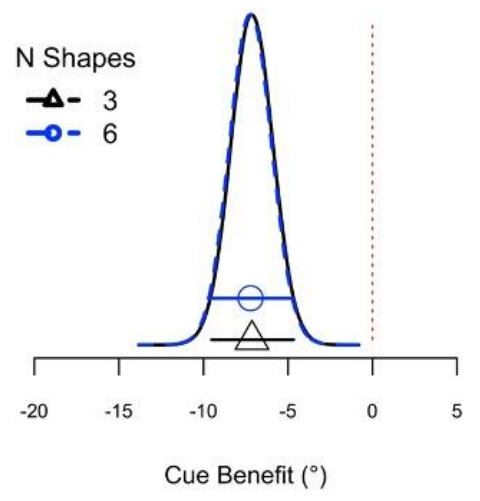


As the shape-cue selected two items in the array, we inspected whether recall error varied as a function of the cued items' spatial contiguity. For the subset of retrocued trials, mean recall error was similar for adjacent and non-adjacent items both for the small cue-set $\left(M_{\text {Adjacent }}=51.36, S D=16.56, M_{\text {NonAdjacent }}=49.99, S D=14.26\right)$ and for the large cue-set $\left(M_{\text {Adjacent }}=51.14, S D=16.49, M_{\text {NonAdjacent }}=50.66, S D=15.75\right)$. A 2 (Adjacent, Non-Adjacent) x 2 (Cue Set-Size: 3, 6) BANOVA having recall error as dependent variable showed evidence against any influence of either spatial contiguity $(\mathrm{BF}$ ratio $=0.20)$ or cue set-size $(\mathrm{BF}$ ratio $=0.14)$. This suggests that, similar to Experiment 3, the feature-cue could be utilized to deploy attention across the whole array. Finally, we also explored whether there were performance differences as a function of single shapes. This analysis is reported in the Supplementary Materials. In sum, we did not find evidence of credible differences.

\section{Main Analyses}

Figure $8 \mathrm{~b}$ presents recall error in Experiment 4 as a function of cue condition and cue set-size (3 vs. 6 items). A 2-way BANOVA showed strong support for the model with cue condition only $\left(\mathrm{BF}=1.08 \times 10^{11}\right)$, and substantial evidence against the effect of cue set-size $(\mathrm{BF}=0.15)$, as well as against their interaction $(\mathrm{BF}=0.19) . \mathrm{MCMC}$ draws from the posterior distribution (see Figure 8c) showed that the retro-cue credibly reduced recall error for both the smaller (3-shape) and larger (6-shape) cue set-size, and the benefits were of similar magnitude. 
Figure 9

Mixture Modeling of Parameter Estimates (Mean and 95\% HDI) for Experiment 4

\section{Experiment 4}
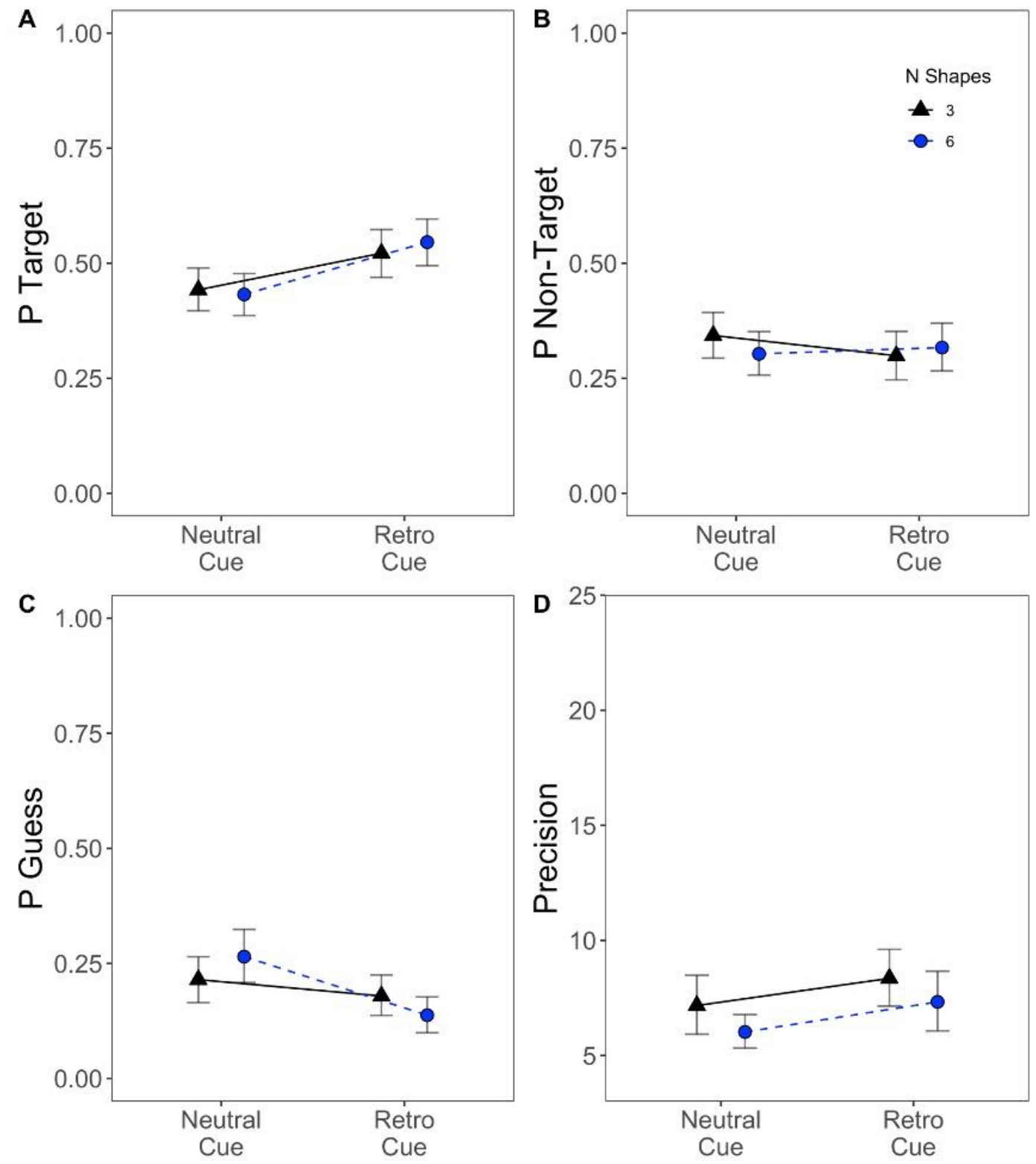
Mixture modeling of this data (see Figure 9 and Table 2) showed that retro-cue was associated with a higher probability of recalling the target item irrespective of the number of cue values within blocks. For the larger cue set-size, this increase in target recalls was due to a credible reduction in guessing. For the smaller cue set-size, the increase in target recalls was due to a mixture of reductions in non-target recalls and guessing which were not credible themselves. Lastly, retro-cues were associated with a slightly larger precision, but this effect was not credible.

\section{Discussion}

Experiment 4 tested whether increasing between-trial variability of shape features detracts resources from proper encoding of the continuous memory feature. Furthermore, Experiment 4 extended our examination of the feature retro-cue effect by employing a different combination of features (color-shape) and a higher set-size (6 items) than previously used, while requiring feature-based selection of two items concurrently. We again obtained consistent feature retro-cue benefits, and these benefits were of similar magnitude irrespective of the variability of the cue values across trials. Critically, we replicated the benefits observed in Experiment 3 regarding concurrent selection of two memory items with no costs in either swap errors or memory precision.

One difference of this to our previous studies is that average recall error was higher than observed in Experiments $1-3$ (i.e., ca. $60^{\circ}-50^{\circ}$ compared to $40^{\circ}-20^{\circ}$ in Experiment 1 and $30^{\circ}-20^{\circ}$ in Experiments 2 and 3, respectively) and probability of recall was around $50 \%$. Note that this study introduced a larger set-size than before, and hence expectedly performance was poorer (with participants being able to recall ca. 3 out 6 items). Importantly, performance was far below the guessing range which is around $90^{\circ}$ 
recall error, arguing against the concern that the results may be biased by an overall floor effect.

Perceptual priming benefits performance in single- as well as conjunction feature tasks (Maljkovic \& Nakayama, 1994; Wang et al., 2005), and higher variance in features on preceding trials impairs performance compared to when variance is low (Michael et al., 2014). Our results suggest that these effects may be specific to the perceptual domain.

\section{Experiment 5}

Experiments 1-4 showed robust and consistent benefits of feature retro-cues that generalized across types of feature cues (colors, orientations, shapes), number of simultaneously cued features (one or two), and change rate in cue values (smaller or larger cue-set). In Experiment 5, we assessed whether feature-based attention could operate on VWM representations when these lacked a distinct spatial context.

As outlined in the introduction, previous research suggested that feature-based selection in VWM may utilize objects' location information as a mediator to access the other item's feature (Pertzov et al., 2013). Evidence for this claim comes from the observation that misreporting one object's feature does not increase errors for the other feature, suggesting their relative independence in memory (Bays et al., 2011; Schneegans \& Bays, 2017), whereas the spatial layout is encoded to VWM and used for retrieval even when irrelevant (Chen \& Wyble, 2015; Kondo \& Saiki, 2012; van Ede et al., 2019). Hence, it is still unresolved whether attentional selection of features can operate independent of spatial context. To test for this possibility, Experiment 5 presented the memoranda sequentially, all at the same location. 
If there is no direct connection between two features of the same object, and access to them requires retrieval of an additional context (e.g., the spatial location or the serial position), then it is possible that feature-cue based memory retrieval may take longer, particularly when this access is made more difficult by the removal of spatial uniqueness. Research with spatial retro-cues has shown that retro-cue benefits take about 300-400 ms to appear and remain stable thereafter (Souza, Rerko, \& Oberauer, 2014; Tanoue \& Berryhill, 2012). To the best of our knowledge, the effect of post-cue time has not been assessed for feature retro-cues. In a pilot study in our lab $(N=20)$ using feature retro-cues and sequential presentation of four memoranda, we did not observe evidence for a retro-cue benefit with a post-cue time of $1 \mathrm{~s}$ (Retro-cue: $M=53.1^{\circ}, 95 \% C I=[52.6$, 59.1]; No-cue, $M=55.8^{\circ}, 95 \% C I=[49.8,56.4]$; $t$-test $\left.\mathrm{BF}=0.77\right)$. Based on this data, we hypothesized that feature retro-cue benefits could take longer to accumulate in the absence of spatial context. Therefore, here we varied the post-cue time along three levels that have been shown to yield robust spatial retro-cue benefits: $0.5,1.0$, and $1.5 \mathrm{~s}$.

\section{Method}

\section{Participants}

A new sample of 51 university students from the Zurich area $\left(M_{\text {Age }}=23.28, S D=\right.$ 3.85, 9 men) completed one session in exchange for 15 Swiss or course credit. As outlined in Experiment 1, we planned to test $N=20$ participants, and to continue data collection until analyses showed evidence for or against an effect (i.e., BF $<0.3$ or $>3$ ), as this approach is feasible when Bayesian estimation is used (Rouder, 2014). In the present study, evidence remained in the ambiguous range until our stopping criteria was reached. 


\section{Procedure}

Figure 10a illustrates the flow of events in a no-cue trial (A), and in the retro-cue trials with varied post-cue delays (B). Materials and stimuli were similar to the ones in Experiment 4. Each trial started with the presentation of a white fixation cross for $1 \mathrm{~s}$. Thereafter a sequence of four colored shapes were presented one-by-one in the center of the screen. The shapes were randomly selected from the pool of six shapes (i.e., circle, square, triangle, halfmoon, cross and star radius $=35$ pixels). Presentation timing was $300 \mathrm{~ms}$ per item with $100 \mathrm{~ms}$ interval between items. After the last item was shown, a blank 1-s retention interval followed. In the no-cue condition, the retention interval was followed by the test display. In the retro-cue conditions, a shape cue (white shape outline in the center of the screen) was shown for $250 \mathrm{~ms}$, followed by either $0.5,1$ or $1.5 \mathrm{~s}$ postcue intervals. Each of the four conditions occurred equally often across trials (hence $75 \%$ of all trials were retro-cue trials). The test display was the same as in previous experiments, except that the probe shape appeared in the screen center. Participants reconstructed the color of the shape from memory by selecting a hue from the color wheel and confirmed their response with a mouse click.

There were 20 practice trials (half with retro-cues). The first four participants completed one session of 400 test trials (100 per design cell). The remaining participants $(n=48)$ completed a longer version with 440 test trials (110 per design cell). At test, after every $1 / 5$ of the trials, participants received cumulative performance feedback in percentage and could make a self-paced break. 


\section{Results}

Figure 10b shows recall error as a function of Cueing (No-Cue, $0.5 \mathrm{~s}, 1 \mathrm{~s}$ and $1.5 \mathrm{~s}$ post-cue delays). Recall error was a bit reduced in the retro-cue conditions compared to the no-cue condition, and a BANOVA showed ambiguous evidence for a cueing effect on performance $(\mathrm{BF}=1.8)^{1}$.

Bayesian $t$-tests between conditions revealed ambiguous evidence for a difference between non-cued condition and delays of 0.5 and $1 \mathrm{~s}(\mathrm{BF}=2.35$ and 2.37, respectively), and some evidence for reduction in recall error when compared to $1.5 \mathrm{~s}$ post-cue delay $(\mathrm{BF}=3.22)$. However, MCMC sampling from posterior distributions indicated decreases in error as credible in all retro-cue conditions compared to the no-cue baseline, although the effects were much smaller compared to previous experiments.

Mixture modelling (see Table 2 and Figure 11) showed a credible increase in target recalls only when comparing the 1.5-s delay retro-cue condition to the no-cue condition. All in all, delaying the test to allow attention to focus on retrieving the items seems to have been only of limited effectivity to improve performance in the task.

\footnotetext{
${ }^{1}$ We monitored the evidence for an effect of cue condition after we collected the first 20 participants. Evidence remained ambiguous as we increased sample size, as shown by continuous reevaluation with addition of every 10 new participants $(N=20: \mathrm{BF}=2.92 ; N=30: \mathrm{BF}=0.37 ; N=40: \mathrm{BF}=0.81)$.
} 


\section{Figure 10}

Experiment 5. Panel (a): Flow of Events in the No-Cue (A) and Retro-Cue (B) Conditions (Note the Variable Delay Durations After Retro-Cue). Panel (b): Recall Error. Panel (c): Posterior of the Retro-Cue Effect Reflecting the Contrast of Each Cue Condition vs. the No-Cue.

(a)

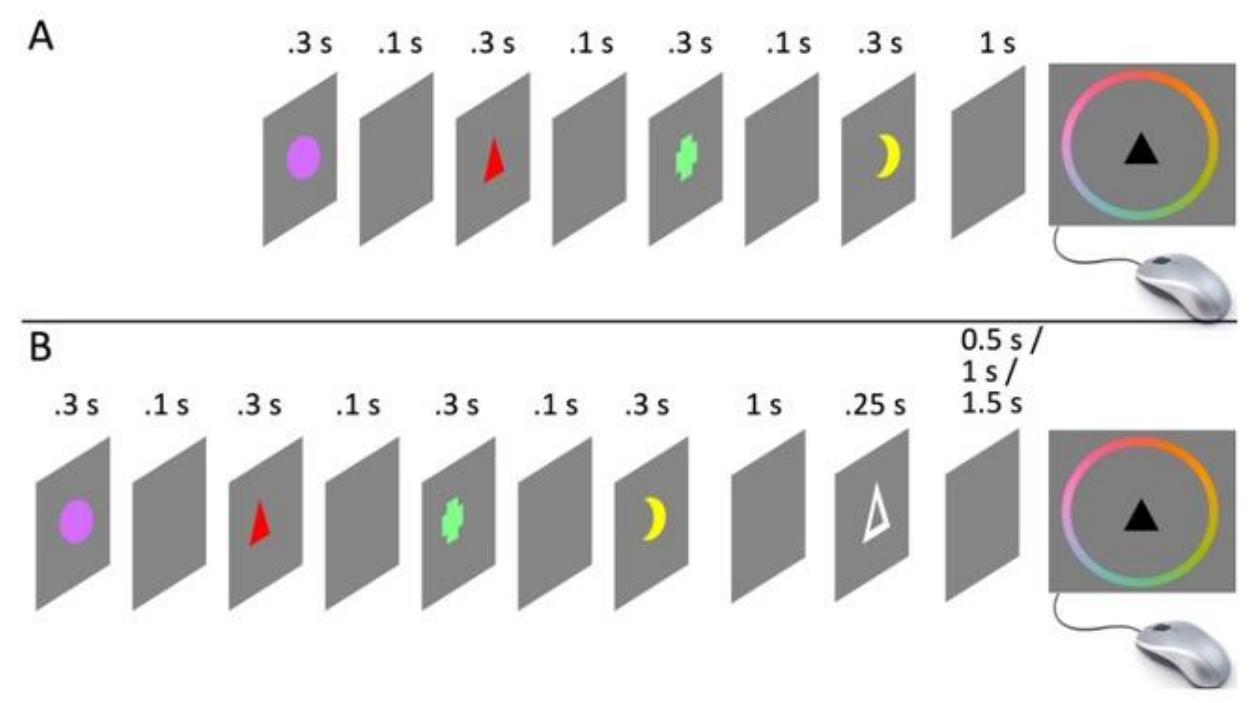

(b)

(c)
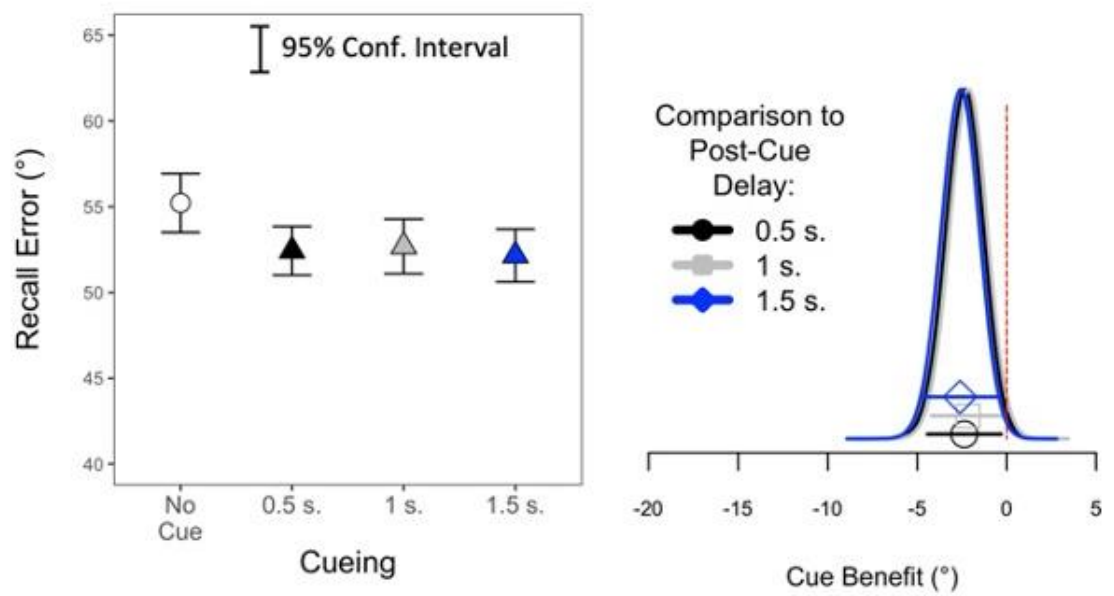


\section{Figure 11}

Mixture Modeling Parameter Estimates (Mean and 95\% HDI) for Experiment 5

\section{Experiment 5}
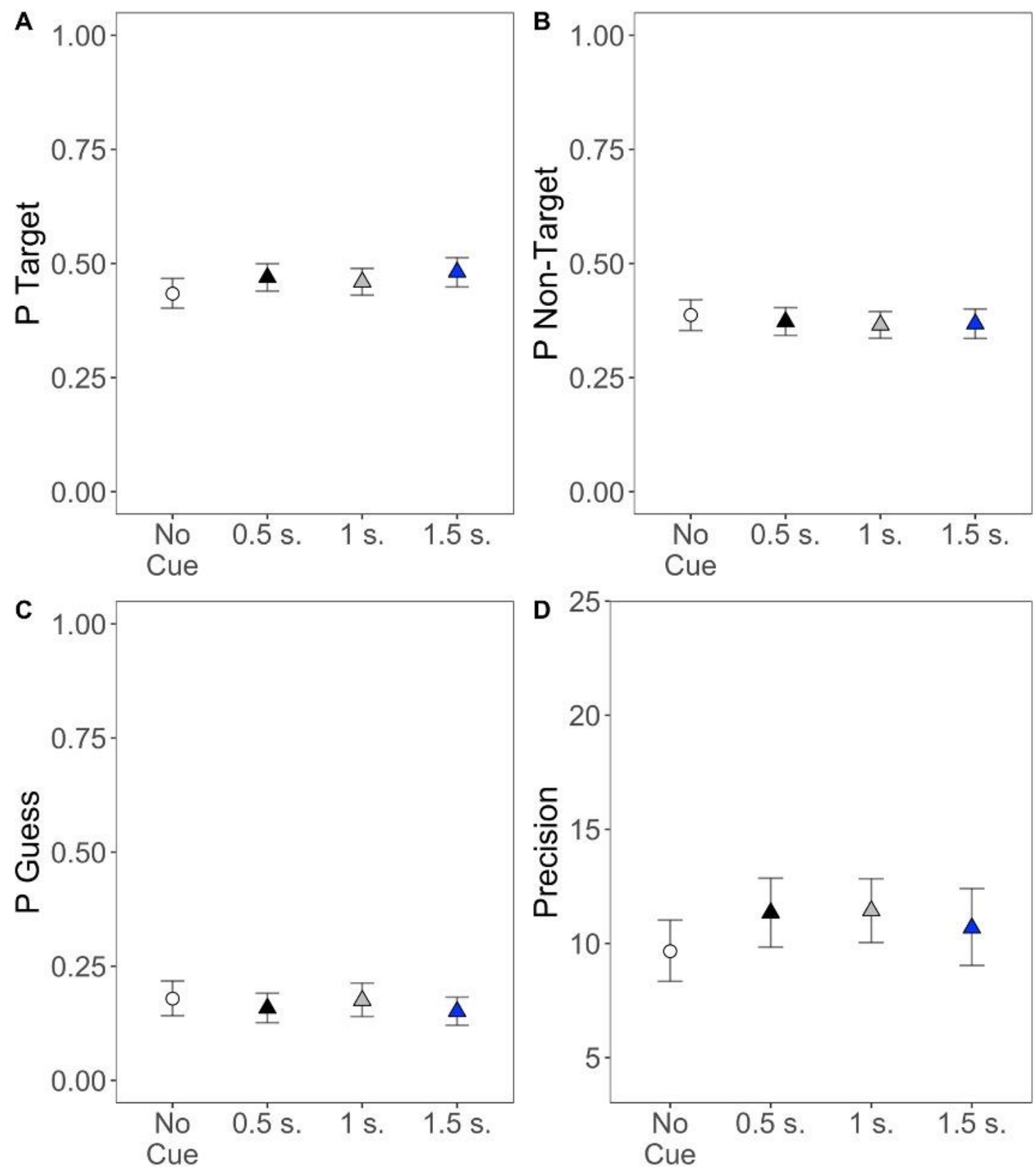
Because items were presented sequentially, we additionally examined item position effect on the cue benefit. A figure with recall error over serial position is presented in the Supplementary Materials. A 4 (Item Position) x 4 (Cueing) BANOVA indicated that the main effect of item position contributed most to differences in recall error $\left(\mathrm{BF}=1.05 \times 10^{187}\right)$. There was no evidence for a main effect of cueing $(\mathrm{BF}=.45)$. There was also decisive evidence against including an interaction $\left(\mathrm{BF}_{\text {Interaction }}=1.2 \mathrm{x}\right.$ $10^{185}$ vs. $\mathrm{BF}_{\text {Main effects }}=4.8 \times 10^{186}$, BF-Ratio $\left.=0.02\right)$.

\section{Discussion}

The results of Experiment 5 showed a diminished feature retro-cue benefit when information was presented sequentially and at the same location. Similar to Experiment 4, average recall error was higher than observed in Experiments 1-3 (i.e., ca. $55^{\circ}-50^{\circ}$ ). This may be due to sequential presentation being more difficult for retrieval in VWM (Pertzov \& Husain, 2014); but, importantly, recall error was far below the floor level of $90^{\circ}$.

Although these results suggest that removing the spatial uniqueness of the items reduced the feature retro-cue benefits, the present study introduced two manipulations at once (i.e., same-location as well as sequential presentation), thus limiting the possibility to attribute changes in performance to one of these factors.

\section{Experiment 6}

Experiment 5 showed small and not fully credible evidence for a feature retro-cue benefit when items were presented in the same spatial location. This suggests that spatial location may mediate or facilitate the use of feature-based attention. However, Experiment 5 also differed from Experiments 1-4 in that items were presented 
sequentially. To determine whether the reduction of the feature retro-cue benefits is related to the sequential presentation or the lack of spatial distinctiveness, Experiment 6 directly contrasted conditions with or without unique spatial context using a sequential presentation mode. In other words, Experiment 6 compared the feature retro-cue benefit in a condition where all items were presented successively in the screen-center to a condition in which sequentially presented memoranda occupied unique locations arranged around an invisible circle (i.e., periphery).

\section{Method}

\section{Participants}

We aimed to recruit a sample of 200 participants for an online study using the Prolific platform. However, due to a problem in the server, we lost data of 12 participants, and hence we obtained a sample of 188 participants. We collected this large sample size to be able to estimate whether there was credible evidence for an interaction between presentation mode (center vs. periphery) and retro-cueing.

We set up the following recruitment criteria: age between 18 and 35 years old, English as first language, and normal color vision and acuity. Participants were invited to take part in a study about learning sequences of colored shapes. They completed the study on their own devices (only computers were allowed) and viewing distance was unconstrained. The study took about 30 min to complete and participants received 4.5 pounds as reimbursement.

The self-reported demographic characteristics of our sample were as follows: $M_{A g e}=26.61, S D=5.08 ; 90$ men; Educational background: $n=1$ Primary school, $n=21$ Secondary/High-school, $n=122$ higher education, $n=39$ Master, $n=5 \mathrm{PhD}$. 


\section{Procedure}

The experiment was programmed using lab.js (Henninger et al., 2020) which is a JavaScript and HTML based tool for programming online experiments. Participants completed a continuous color reproduction task with colored shapes similar to the one described for Experiment 4: a sequence of four colored shapes (size ca. 55 pixels) was presented for study at a rate of one shape every $400 \mathrm{~ms}$ (300 ms onscreen, $100 \mathrm{~ms}$ offscreen, see Figure 12a). In the Center-presentation condition, all items were presented successively in screen-center. In contrast, in the Periphery-presentation condition, the memoranda appeared successively in four fixed periphery locations (top, right, bottom, and left) placed on an invisible circle (radius $=120$ pixels). The Center- and Peripherytrials were randomly intermixed. Half of the trials in the center and periphery conditions consisted of a short no-cue baseline condition in which after a retention interval of $1 \mathrm{~s}$ following the last item, the test display was presented. The other half of the trials consisted of retro-cue trials: the outline of the to-be-tested shape was presented in the screen-center for $250 \mathrm{~ms}$, followed by a 1-s post-cue interval, at the end of which the test display appeared. The test display consisted of a black shape presented on the screen center surrounded by a color wheel. The test display was identical in all conditions. Participants clicked on the color on the wheel that they believed the shape had appeared in. This was followed by a 1-s inter trial interval. Participants completed 12 practice (nocue: 4 , retro-cue: 8 ) and 208 test trials. 


\section{Figure 12}

Panel (a) Presents the Flow of Events in Experiment 6: Stimulus Presentation (A),

Maintenance Interval (B), and Test (C). Panel (b) Displays Recall Error, and Panel (c) the MCMC Posterior of the Retro-Cue Effect per Condition.

(a)
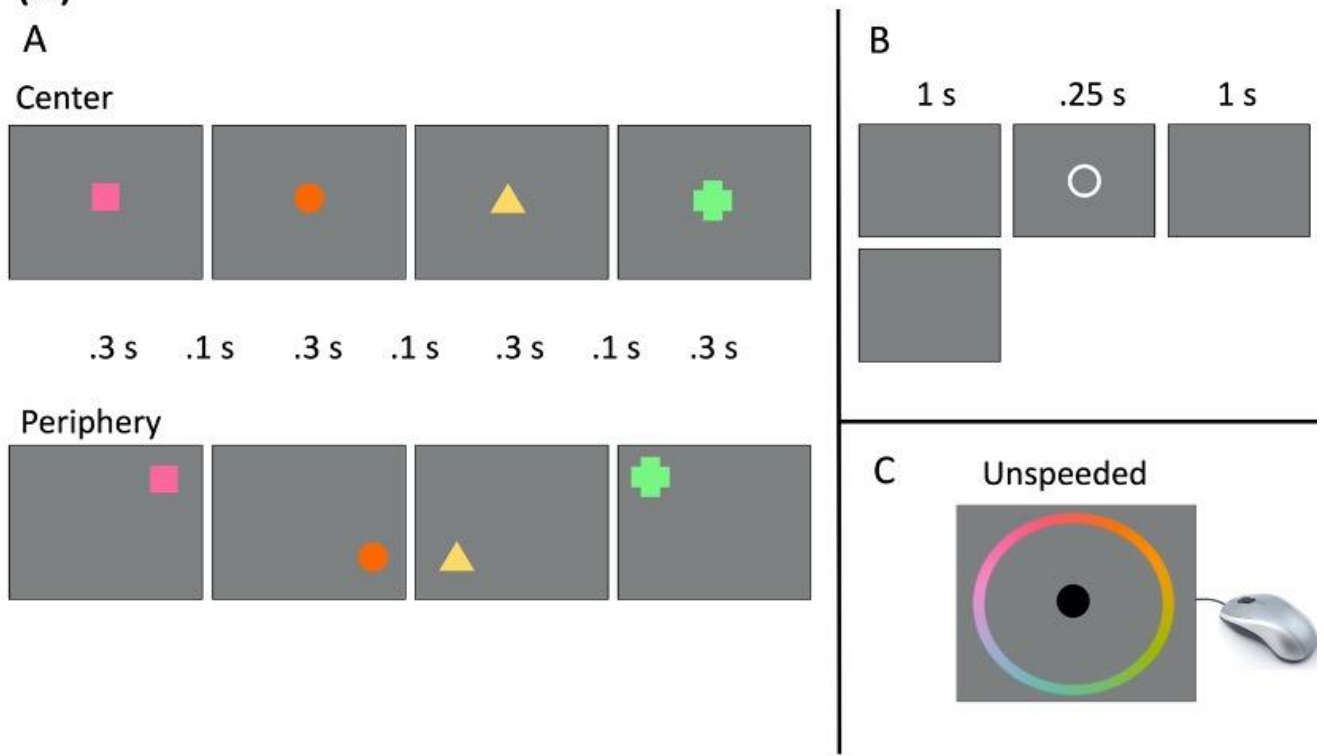

(b)

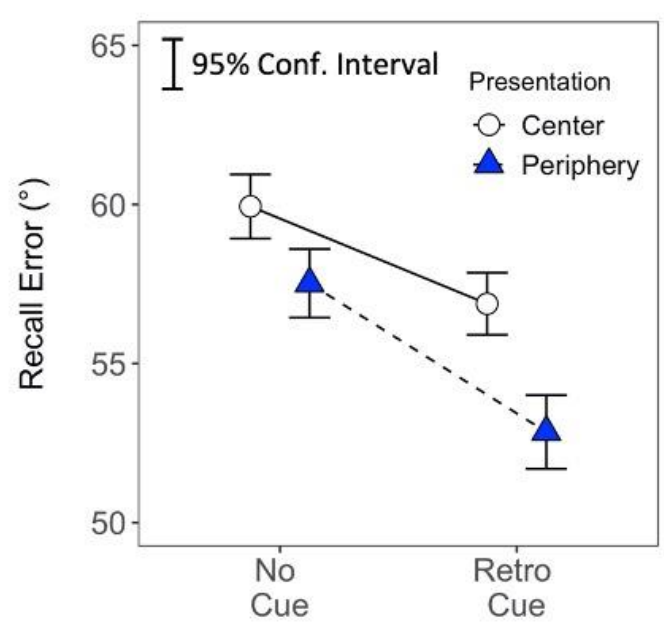

(c)

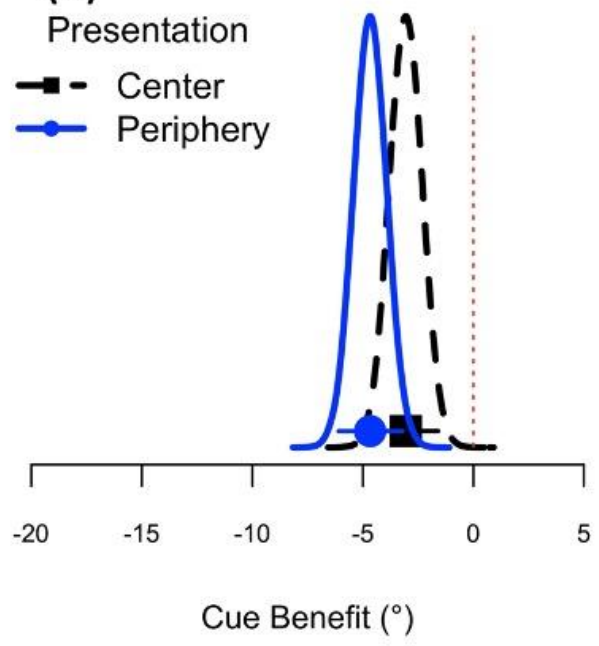




\section{Results}

Figure $12 \mathrm{~b}$ shows recall error as a function of Cueing (No-Cue, Retro-Cue) and Presentation (Center, Periphery). A $2 \times 2$ BANOVA on recall error revealed credible main effects of both factors (model $\left.\mathrm{BF}=1.85 \times 10^{15}\right)$. Partitioning the evidence in $\mathrm{BF}$ ratios identified strong support for the factor of Presentation $\left(\mathrm{BF}\right.$ ratio $\left.=2.37 \times 10^{6}\right)$, as well as Cueing $\left(\mathrm{BF}\right.$ ratio $\left.=3.08 \times 10^{9}\right)$, whereas evidence against the interaction almost reached credibility $(\mathrm{BF}$ ratio $=0.34)$. Follow-up $t$-tests indicated credible evidence for reductions in recall error in the Periphery condition $\left(\mathrm{BF}=2.23 \times 10^{5}\right)$ and Center condition ( $\mathrm{BF}=972)$. MCMC sampling (depicted in Figure 12c) showed that cueing benefit in the periphery condition was larger, but both conditions' HDIs did not include zero, indicating credible benefits [Center: $M=-3.05,95 \%$ HDI: $-4.509,-1.61]$, Periphery: $M=-4.66,95 \%$ HDI: $-6.11,-3.22]$. Hence with the large sample size in Experiment 6, the smaller retro-cue benefit observed when items were presented in the center of the screen could be measured credibly. When items appeared in a unique spatial context the benefits were larger and hence easier to measure.

To estimate when feature retro-cue benefits became credible in each condition, we conducted a sequential Bayesian $t$-test analysis in JASP (JASP Team, 2020). In this analysis, the evidence for an effect is recomputed as each new participant is entered in the sample. As one can see in Figure 13, credible evidence $(\mathrm{BF}>10)$ was slower to accumulate in the Center condition than in the Periphery condition. For the Center condition, a BF >10 was only obtained when $\mathrm{N}>110$, whereas the same threshold was reached with less than half of this sample-size in the Periphery condition. 


\section{Figure 13}

Sequential Evidence Analysis in Experiment 6.

A

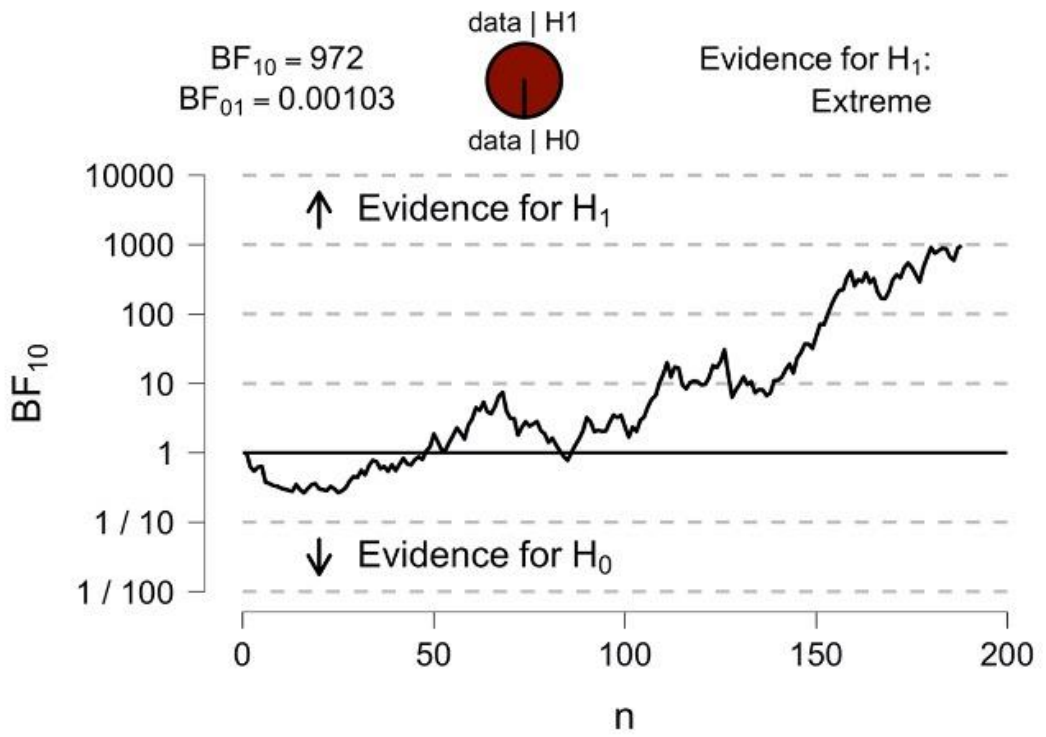

B

\section{Periphery}

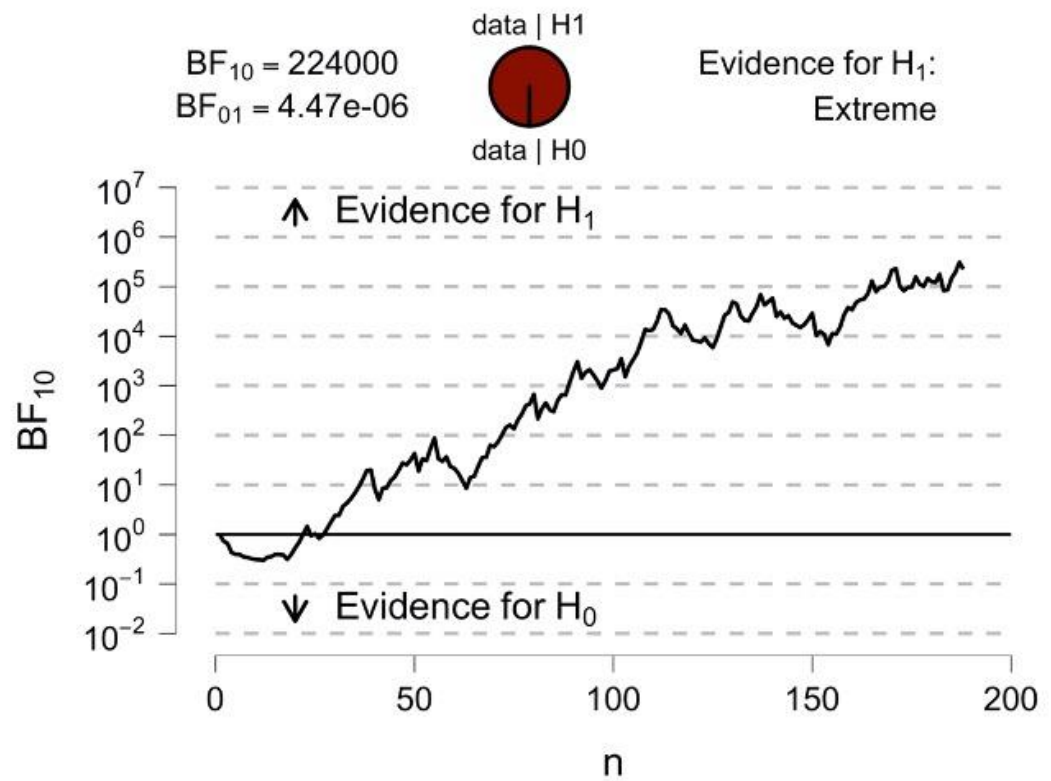


To parallel Experiment 5, we also analyzed cue benefit as a function of items' serial input position. A figure with performance over serial position is available in the Supplementary Materials. A 4 (Input Position) x 2 (Presentation: Center, Periphery) BANOVA on cue benefit (i.e., the difference between recall error in the no-cue and retrocue condition) revealed strong evidence against the effect of items' serial position (BF ratio $=0.008)$, as well as against the role of presentation mode $(\mathrm{BF}$ ratio $=0.26)$.

Mixture modeling (visualized in Figure 14) revealed credibly higher probability of target responses with cueing both in the Center and Periphery presentation modes, as well as credible reductions in non-target responses with cueing in the peripheral condition. Together, these results indicate that even when spatial distinctiveness is not given, feature-based attention can operate on representations in VWM, but this facility is more limited in comparison to when both feature- and spatial-contexts are available. In sum, the evidence indicates that both spatial- and feature-based attention in VWM can lead to memory boosts, and that these benefits may be additive.

\section{Discussion}

Experiment 6 provided evidence that when a unique spatial context was associated with each memory representation, robust benefits from feature retro-cues accrued even with a sequential presentation mode. In the absence of spatial uniqueness, participants were still able to draw on the cue information to improve VWM accuracy, albeit to a reduced extent.

Together, Experiments 5 and 6 indicate that without a unique spatial context, feature-based access to VWM representations is not lost, but the effect is harder to measure compared to when spatial context is given, and requires a large sample (approx. 
$N=100)$ to emerge. One possibility to explain this pattern of results is the notion that adding more contexts for retrieval - i.e., spatial- as well as temporal - benefits memory access.

\section{Figure 14}

Mixture Modeling Parameter Estimates (Mean and 95\% HDI) for Experiment 6

\section{Experiment 6}
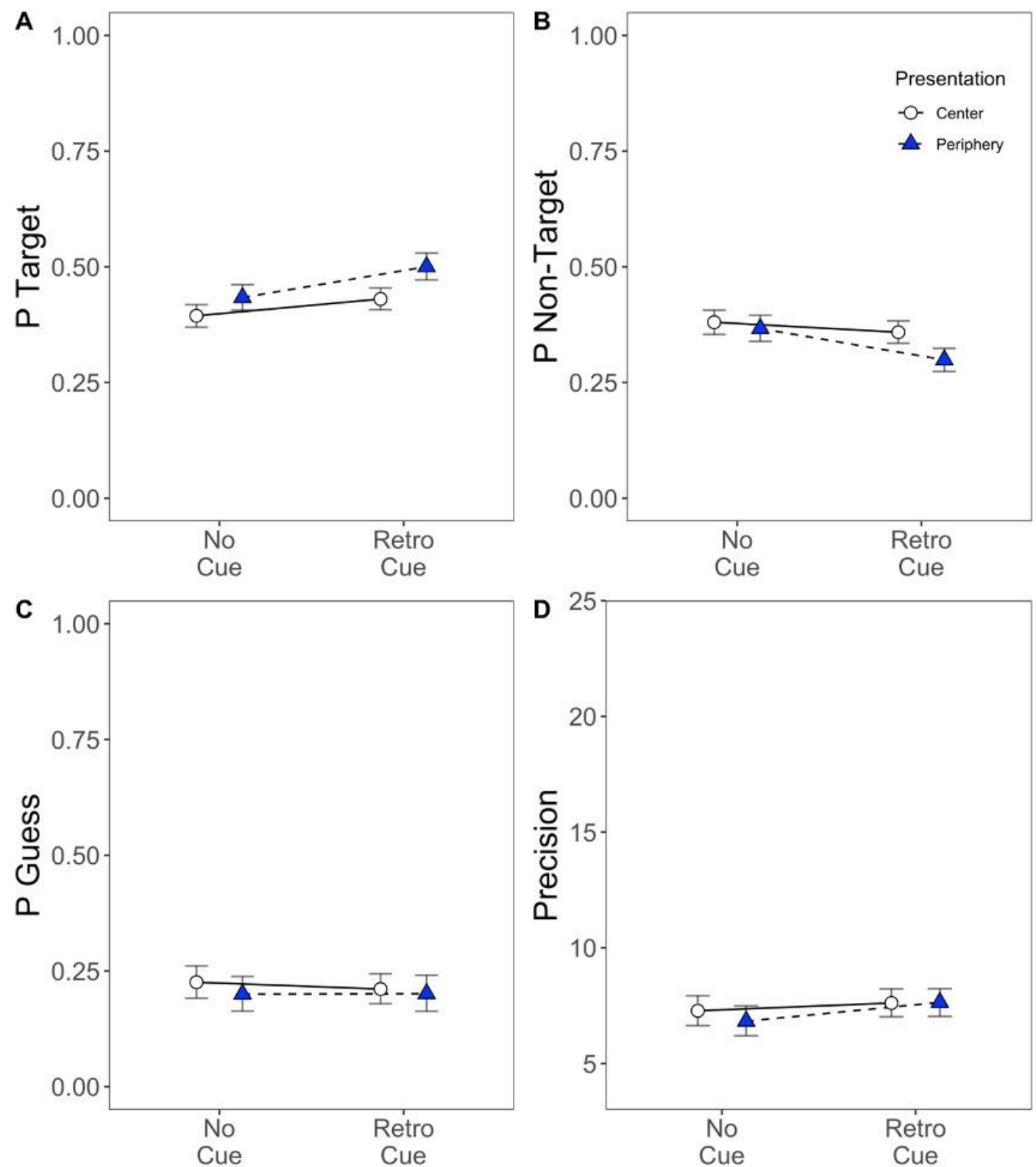


\section{Overview of Retro-Cue Effects Across All Experiments}

As shown in Figure 15, feature retro-cues produced mostly credible reductions in recall error. These benefits were largest in Experiment 1 in which most trials presented retro-cues (70\%), and were a bit reduced when retro-cues appeared in only $50 \%$ of the trials (Experiments 1, 2, 3, 4 and 6). Critically, retro-cue benefits were smallest when the memoranda lacked spatial distinctiveness (Experiments 5 and 6, Center condition).

Figure 16 presents an overview of mixture modeling results. Across all experiments, feature-cueing benefits were most consistent for the probability of target recall. Overall, this was due to reductions in both non-target recall and guessing, with changes in these parameters being hardly credible by themselves. Similarly, across all experiments, memory precision tended to be higher following cueing, although these estimates were usually not credible. 


\section{Figure 15}

Overview of Reduction in Error in Degrees (Panel A) and Percent (Panel B) Against

Baseline Conditions Across Experiments 1-6. Shapes and Fill Vary for Visual

Disambiguation Between Experiments

\section{Overview All Experiments}

A

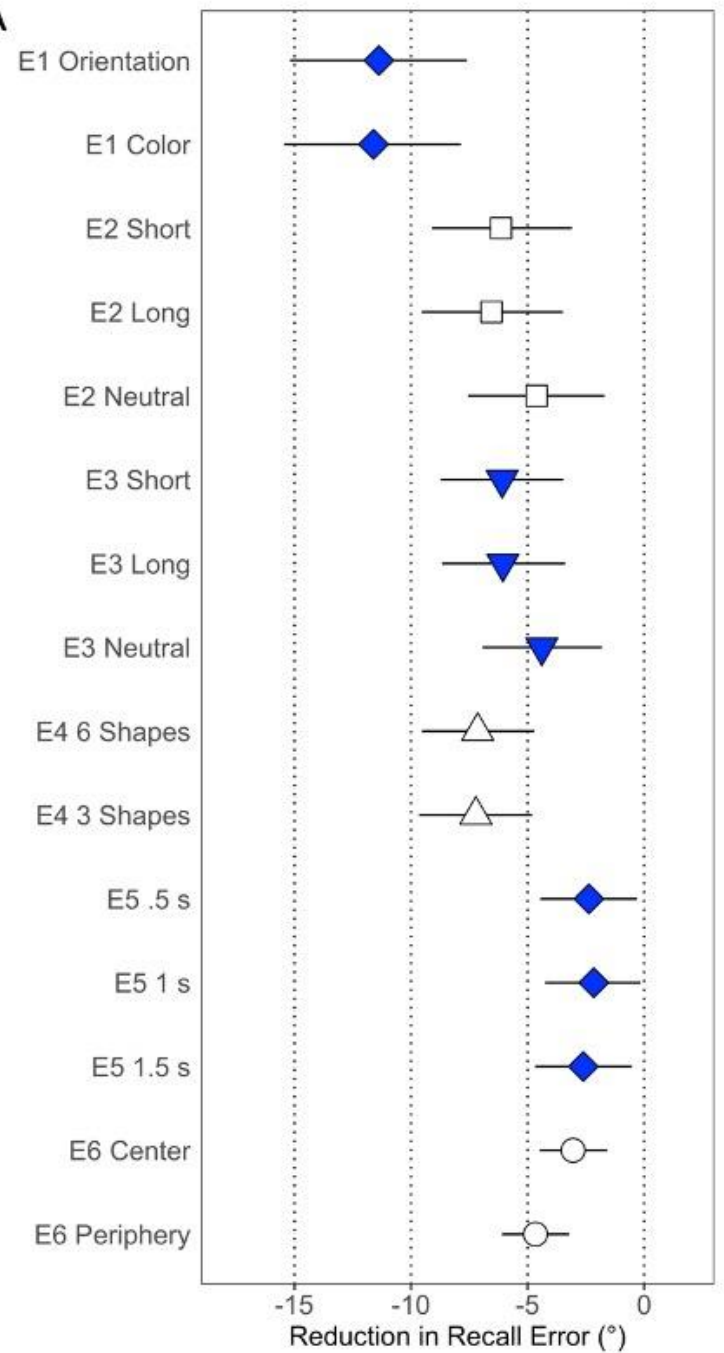

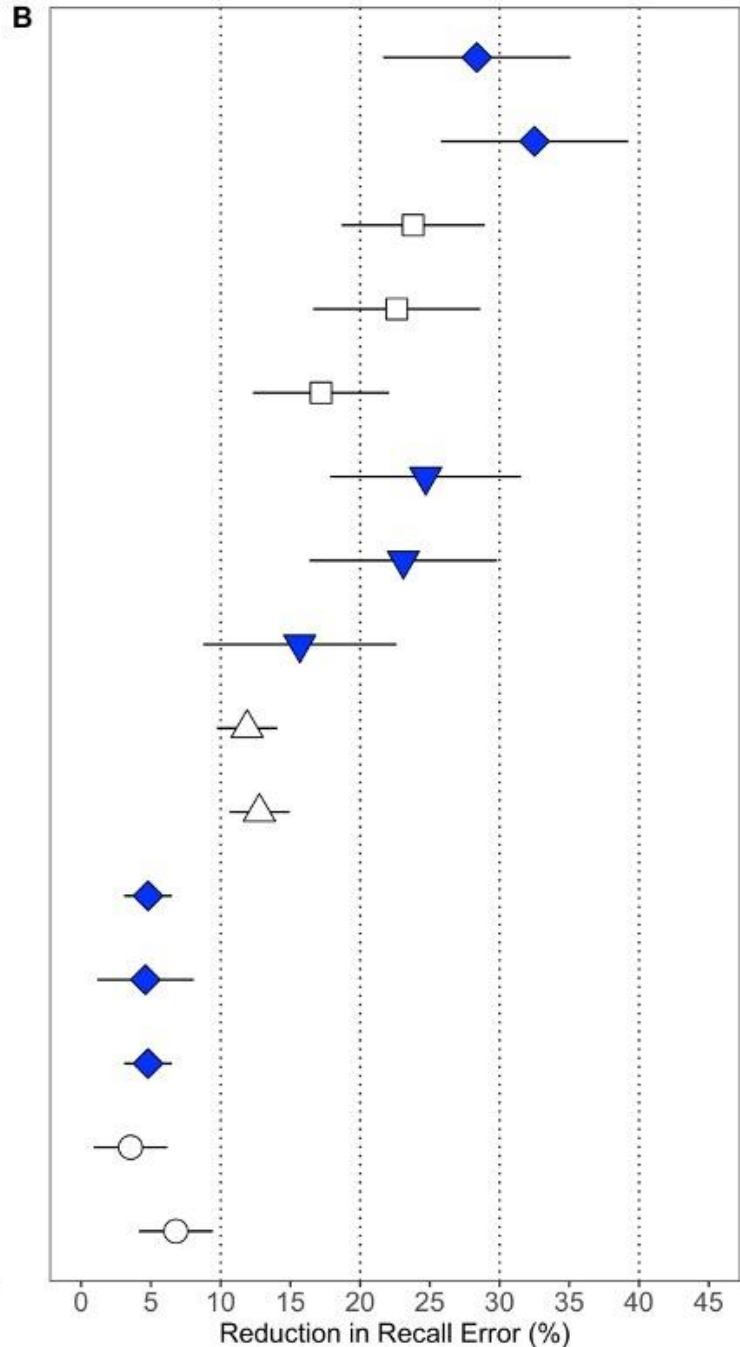

Note. The Error Bars are the MCMC Chains' 95\% Highest Density Intervals. 


\section{Figure 16}

Overview of the Absolute Feature Retro-Cue Effect on Mixture Modeling Parameter

Estimates (Mean and 95\% HDI) across Experiments 1-6. Filled Shapes Denote Credible

Effects

\section{Overview all Experiments}

A

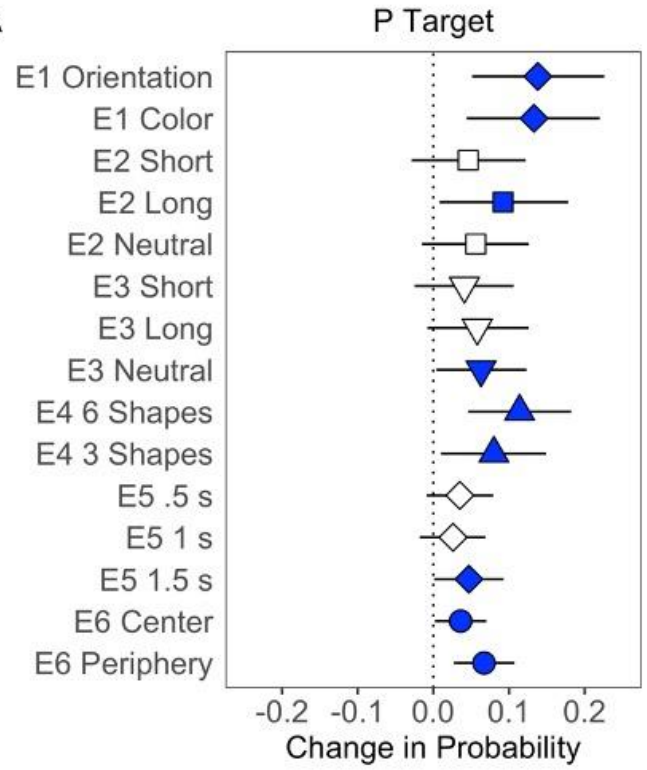

C

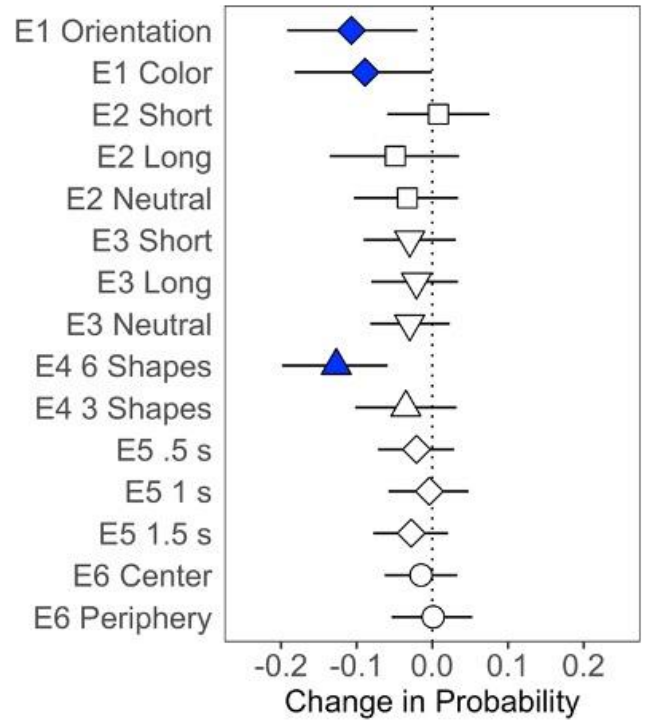

B

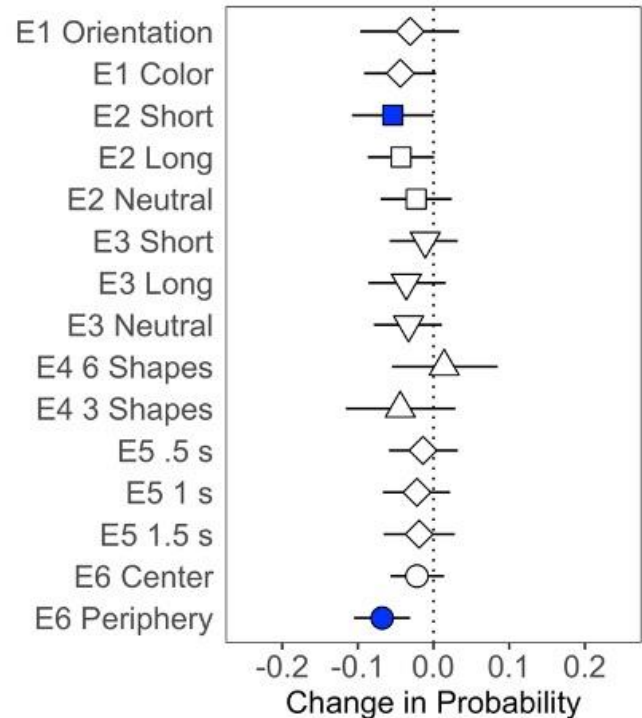

D

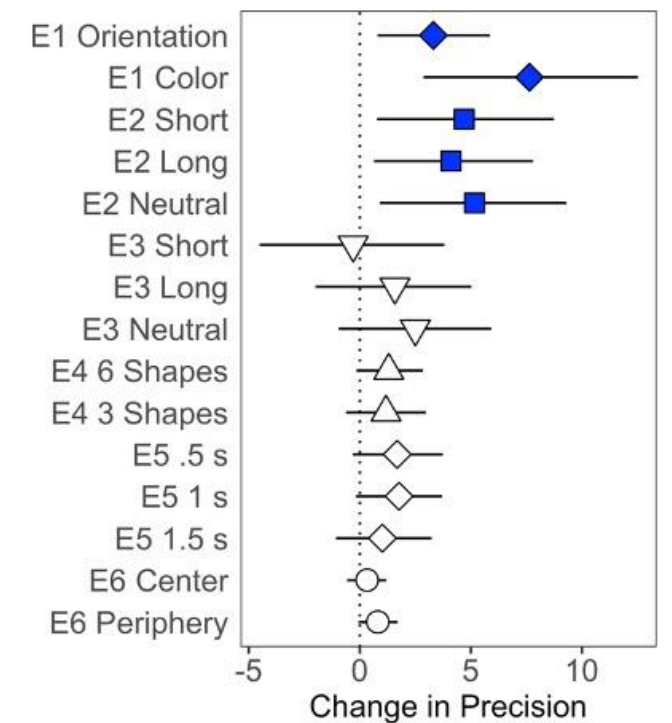




\section{General Discussion}

In this study, we tested the scope of the ability to attend to features in VWM.

Feature retro-cue benefits were robust to several manipulations, such as the use of different cue feature dimensions (colors, orientations, shapes) and compared to different baselines that controlled for time-based forgetting (i.e., short no-cue condition) and interference induced by the cue itself (long no-cue vs. neutral-cue condition). Furthermore, feature retro-cues were similarly effective to select a single item or two items spread across different locations in the array, and also when the number of possible cue values was varied across trials, which could challenge attention guidance.

All experiments used a continuous delayed estimation task that allows estimation of both the quantity and quality of information in memory via the use of mixture models. Across experiments, mixture modelling revealed more consistent feature retro-cue benefits for the accessibility of information in memory (PTarget parameter), and only sometimes for memory precision. Across the six experiments, there was only one condition that was associated with somewhat smaller feature retro-cue effects, namely when the memory array lacked spatial distinctiveness.

\section{Time-Based Forgetting and Perceptual Interference}

Experiments 2 and 3 assessed whether feature retro-cue benefits are robust when this condition is contrasted to baselines that control for protection from time-based forgetting and from perceptual interference. In our review of the literature, we noticed that most studies compared feature retro-cues against a matched neutral-cue condition (Barth \& Schneider, 2018; Heuer et al., 2016; Heuer \& Schubö, 2016; Kalogeropoulou et al., 2017; Li \& Saiki, 2015). Given that neutral-cue conditions may yield lower 
performance than no-cue conditions (Murray et al., 2013), we hypothesized that presentation of the neutral cue could incur interference, and that the feature retro-cue condition could protect from this cost. Here we ruled out this alternative explanation: Feature retro-cues were similarly beneficial when compared to trials with or without neutral-cues. We also ruled out the possibility that feature retro-cues help by protecting against time-based forgetting over short intervals: Feature retro-cue benefits were credible when compared to a short no-cue baseline. These findings are similar to the ones observed for spatial retro-cues, suggesting that these retro-cues may operate in similar ways to prioritize information in VWM.

\section{Feature Selection: Multiple Items in the Focus of Attention}

A prominent property of feature-based attention is that it can be deployed broadly over space (Carrasco, 2011; Gledhill et al., 2015; Maunsell \& Treue, 2006; McAdams \& Maunsell, 2000; Saenz et al., 2002; Schledde et al., 2016; Treue \& Trujillo, 1999). Feature retro-cues were previously shown to afford selecting more than one item in VWM simultaneously in a change detection task (Heuer \& Schubö, 2016). In Experiments 3 and 4, we replicated this finding in a continuous reproduction task using colors and shapes as cues to retrieve continuous orientations and colors from VWM, respectively, and with set-sizes near and above traditional estimates of VWM capacity. Selection of multiple items concurrently seems rather specific to feature attention. There are mixed reports in the literature whether spatial retro-cues can select multiple items at the same time. For example, Makovski and Jiang (2007) reported that spatial retro-cues to one location improved memory in change detection task, but this effect did not persist when two or three out of six elements were retro-cued. Another study found that 
concurrent benefit for change detection of two cued colors was possible when these were located in different hemifields (i.e., on the left and right side of the display), but not when both cued items occurred within the same hemifield (Delvenne \& Holt, 2012). Similarly, Schneider et al. (2015) presented symmetrical arrays of four colored discs and retro-cued either the left or right side of the screen (which contained two items each), observing higher change detection accuracy in the cue condition. There is also evidence that retrocueing an entire set of three items improves change detection when the whole set is shown at test (Matsukura \& Vecera, 2015; Williams \& Woodman, 2012).

Some studies indicate that parallel selection of several items in VWM may depend on their spatial distance. For example, Souza et al. (2018) investigated the spatial precision of selection in perceptual and internal attention using pre-cues and retro-cues. They found that invalidly testing one of the non-cued items produced costs, except for items spatially close to the cued one which were spared from costs. These findings indicate that spatial attention selection is spatially imprecise, and it can select more items as long as they are in the vicinity of the focused location.

Here, we obtained a benefit when the cue referred to two items in the array, and this benefit did not vary with the spatial distance between the cued items, thereby extending the results of Heuer and Schubö (2016) to a delayed estimation task. Joint mixture modelling of data from Experiments 2 and 3 further suggested that simultaneous selection of two items did not result in weaker representations than when the cue tagged a single item: There was no increase in misbinding errors, indicating that parallel selection of two VWM representations did not lead to false mapping of features, and there was no 
cost on precision, indicating that the two cued items' feature values were not approximated. The same pattern of findings was replicated in Experiment 4.

It is still unclear what limits the simultaneous selection of multiple items in the focus of attention. Some models have delineated the focus of attention in VWM as a device that is functionally constrained to the selection of a single representation, unless memoranda are highly distinct (Oberauer \& Lin, 2017; Rerko \& Oberauer, 2013).

Selection into the focus of attention would be constrained to a single element in situations in which confusions between items are likely to occur, but it should be possible when the information is sufficiently distinct. In the spatial retro-cueing task, selection of multiple items is undermined when it occurs concurrently (Makovski \& Jiang, 2007), but not if it occurs sequentially: Several studies have demonstrated benefits to multiple sequentially cued items (Rerko \& Oberauer, 2013; Souza et al., 2015; Souza \& Oberauer, 2017). This shows that the ability to keep more than one item in a privileged status in VWM is more flexible than previously assumed. Concurrent selection of multiple items through feature retro-cues defies the assumption that cross-talk between similar items would constrain multiple selection into the focus of attention. This rather points to a distinction in the use of spatial vs. feature-based attention as a selection device more than whether multiple items can be concurrently accessed in VWM.

\section{Larger Cue Variability, Less VWM Capacity Left?}

We asked whether high variability in cue values may constrain the ability to use them for retrieval in VWM. Previous studies with feature retro-cues used small sets of memory items with only two to four cue-feature values that repeated on every trial (Barth \& Schneider, 2018; Heuer \& Schubö, 2016; Kalogeropoulou et al., 2017; Li \& Saiki, 
2015). Repeating visual stimuli has been found to encumber VWM performance due to proactive interference by information from preceding trials (Endress \& Potter, 2014), and this effect is exacerbated when items share the same spatial location (Makovski, 2016; Makovski \& Jiang, 2008). In Experiment 4, we extended the number of cue feature values to six and let them vary across trials, while keeping the number of features within the trial constant. The results showed that with up to six unique feature values, retro-cue effects were of the same magnitude as when cues were selected from a fixed set of three shapes that reoccurred on every trial. This suggests that feature-based attentional selection can be efficient even under cue uncertainty and hence varying these values did not consume more VWM capacity.

\section{Feature Retro-Cue Effects and Spatial Layout}

In Experiment 5, we removed the spatial distinctiveness of the memory items by showing all colored shapes sequentially in the screen-center, and the retro-cue trial varied the post-cue time thereby increasing the opportunity to successfully use the cue, even if access to the cued features required indirect access to the temporal context of the item. Removing the spatial context diminished feature retro-cue benefits: Evidence for a benefit remained ambiguous although we collected a considerable number of participants $(N=51)$. Experiment 6 directly compared the role of unique vs. shared location when memoranda were presented sequentially in a very large sample of participants $(N=188)$. Retro-cue benefits were larger and easier to detect when items had a unique spatial context, suggesting that spatial distinctiveness seems to contribute to the cueing benefit. However, even at shared locations, the ability to retrieve feature information was still present, albeit reduced. 
One may wonder if presenting objects at the same location may have acted as a mask, interfering with consolidation - i.e., the transfer of perceptual representations into a robust state in VWM (Ricker \& Hardman, 2017). These authors concluded that consolidation of a visual representation incurs an attentional blink - a situation when the subsequent stimulus cannot receive enough attention for proper processing when presented within up to $500 \mathrm{~ms}$ from the onset of the preceding item, as the preceding item's consolidation is still ongoing. Although our stimuli followed each other within 400 ms, serial position curves (visualized in the supplementary materials which are available on the OSF) indicated a strong recency effect. This is inconsistent with an attentional blink effect: This being the case, accuracy for the first item in the sequence should have been the highest. That accuracy improved toward the end of the sequence suggests that failed consolidation is unlikely to explain the diminished cue-guidance benefit in this experiment.

Rather, it may be the case that context - spatial or temporal - is vital to bind features into a representation. This is suggested by Schneegans' et al. (2018) results, who presented three colored shapes in screen center for $400 \mathrm{~ms}$ each and allowed ample time (600 ms) between the stimuli (hence, consolidation was not constrained). At test, observers reported both color and shape, cued by the item's ordinal position. Alternatively, they reported color and ordinal position, cued by the item's shape. Schneegans et al. (2018) found that color and shape were unlikely to be stored as bound features, as erroneous color reports did not increase errors in shape reports. In other words, errors on the feature dimensions were independent of each other, suggesting that these were not inextricably bound to each other. The role of ordinal position, however, was different: When an 
incorrect ordinal position was reported (cued by the shape), color reports tended to correspond to the wrongly identified ordinal position, indicating that color and temporal position information was stored jointly. This is corroborated by Pertzov and Husain's (2014) study, in which orientations were presented simultaneously or sequentially either at the same or different spatial locations. For both simultaneous and sequential presentation, they observed higher probability of misbinding errors when the memoranda had shared the spatial location. Misbindings were exclusively caused by the shared location: When the objects shared the same color, probability of non-target report did not increase. Pertzov and Husain (2014) suggested that spatial location plays a role in enabling the binding of features or accessing these bindings in memory. One further possibility - closely related to the notion of spatial context - is proactive interference, known to impair VWM performance in experimental designs using repeating stimuli across trials (Endress \& Potter, 2014), and being especially impeding when visual items' locations overlap (Makovski, 2016; Makovski \& Jiang, 2008).

\section{Feature-Based Retro-Cues vs. Feature-Dimension Cues}

Finally, here we assessed the scope of feature-based selection of one or two items in VWM based on a single feature value. Recently, studies have also showed that retrocues can be used to guide attention to one feature dimension over another in a manner that extends to all feature values and all items in VWM (Hajonides et al., 2019; Niklaus et al., 2017; Park et al., 2017; Ye et al., 2016). For example, after encoding a set of items that vary both in color and orientation, participants can benefit from a retro-cue guiding attention to the color dimension (but see Maniglia \& Souza, 2020). This indicates that participants can give higher weight to the cued feature dimension or, alternatively, drop 
the uncued dimension from VWM freeing capacity. It is so far unclear to what extent these two types of feature attention selection are related. In the one case, participants focus on one particular feature value (e.g., red), whereas in the other they focus on all values (i.e., color in general). Future studies are needed to fully uncover the similarities and differences between spatial and feature-based object selection, on the one hand, and feature-dimension selection, on the other hand.

\section{Conclusion}

Retrospective attention to visual features, similar to attention to spatial locations, is a powerful and versatile mechanism to improve VWM performance. Retrieval can be guided by a diverse set of feature-cues (color, orientation, shape) enabling the retrieval of the other feature of the same object. Here we showed that feature retro-cue benefits are not explained by protection from time-based forgetting over short intervals nor by perceptual interference yielded by the cueing procedure. A hallmark of feature-based attention in perception as well as in VWM is the ability to extend its effects across space. We replicated this property with a delayed estimation task by showing that two items could be selected simultaneously, producing benefits that were not accompanied by hidden costs to precision or confusion between concurrently selected items. Although feature-based attention may select elements across multiple locations, its use seems to be facilitated by spatial properties: When VWM representations lacked a distinct spatial context, benefits of feature-based attention took longer to emerge. However, that these benefits persisted even without spatial context indicates that feature-based attention is separate from spatial attention, although its operation is facilitated when spatial selection is viable. 


\section{References}

Alvarez, G. A., \& Cavanagh, P. (2004). The Capacity of Visual Short-Term Memory is Set Both by Visual Information Load and by Number of Objects. Psychological Science, 15(2), 106-111. https://doi.org/10.1111/j.0963-7214.2004.01502006.x

Baldassi, S., \& Verghese, P. (2005). Attention to locations and features: Different topdown modulation of detector weights. Journal of Vision, 5(6), 7-7. https://doi.org/10.1167/5.6.7

Barth, A., \& Schneider, D. (2018). Manipulating the focus of attention in working memory: Evidence for a protection of multiple items against perceptual interference. Psychophysiology, 55(7), e13062. https://doi.org/10.1111/psyp.13062

Bays, P. M., Catalao, R. F. G., \& Husain, M. (2009). The precision of visual working memory is set by allocation of a shared resource. Journal of Vision, 9(10), 7-7. https://doi.org/10.1167/9.10.7

Bays, P. M., Wu, E. Y., \& Husain, M. (2011). Storage and binding of object features in visual working memory. Neuropsychologia, 49(6), 1622-1631. https://doi.org/10.1016/j.neuropsychologia.2010.12.023

Brainard, D. H. (1997). The psychophysics toolbox. Spatial Vision, 10(4), 433-436.

Carrasco, M. (2011). Visual attention: The past 25 years. Vision Research, 51(13), 14841525. https://doi.org/10.1016/j.visres.2011.04.012

Chen, H., \& Wyble, B. (2015). The location but not the attributes of visual cues are automatically encoded into working memory. Vision Research, 107, 76-85. https://doi.org/10.1016/j.visres.2014.11.010 
Cowan, N. (2010). The Magical Mystery Four: How Is Working Memory Capacity Limited, and Why? Current Directions in Psychological Science, 19(1), 51-57. https://doi.org/10.1177/0963721409359277

Delvenne, Jean-François, Cleeremans, A., \& Laloyaux, C. (2010). Feature Bindings Are Maintained in Visual Short-Term Memory Without Sustained Focused Attention. Experimental Psychology, 57(2), 108-116. https://doi.org/10.1027/1618$3169 / \mathrm{a} 000014$

Delvenne, Jean-Francois, \& Holt, J. L. (2012). Splitting attention across the two visual fields in visual short-term memory. Cognition, 122(2), 258-263. https://doi.org/10.1016/j.cognition.2011.10.015

Endress, A. D., \& Potter, M. C. (2014). Large capacity temporary visual memory. Journal of Experimental Psychology: General, 143(2), 548-565. https://doi.org/10.1037/a0033934

Fougnie, D., Asplund, C. L., \& Marois, R. (2010). What are the units of storage in visual working memory? Journal of Vision, 10(12), 27-27. https://doi.org/10.1167/10.12.27

Gilchrist, A. L., Duarte, A., \& Verhaeghen, P. (2016). Retrospective cues based on object features improve visual working memory performance in older adults. Aging, Neuropsychology, and Cognition, 23(2), 184-195. https://doi.org/10.1080/13825585.2015.1069253

Gledhill, D., Grimsen, C., Fahle, M., \& Wegener, D. (2015). Human feature-based attention consists of two distinct spatiotemporal processes. Journal of Vision, 15(8), 1-17. https://doi.org/10.1167/15.8.8 
Gressmann, M., \& Janczyk, M. (2016). The (Un)Clear Effects of Invalid Retro-Cues. Frontiers in Psychology, 7. https://doi.org/10.3389/fpsyg.2016.00244

Griffin, I. C., \& Nobre, A. C. (2003). Orienting Attention to Locations in Internal Representations. Journal of Cognitive Neuroscience, 15(8), 1176-1194. https://doi.org/10.1162/089892903322598139

Hajonides, J. E., Ede, F. van, Stokes, M. G., \& Nobre, A. C. (2019). Comparing the prioritisation of items and feature-dimensions in visual working memory. BioRxiv, 863191. https://doi.org/10.1101/863191

Hardman, K. O., \& Cowan, N. (2015). Remembering complex objects in visual working memory: Do capacity limits restrict objects or features? Journal of Experimental Psychology: Learning, Memory, and Cognition, 41(2), 325-347. https://doi.org/10.1037/xlm0000031

Henninger, F., Shevchenko, Y., Mertens, U., Kieslich, P. J., \& Hilbig, B. E. (2020). lab.js: A free, open, online experiment builder. Zenodo. https://doi.org/10.5281/zenodo.3767907

Heuer, A., \& Schubö, A. (2016). Feature-based and spatial attentional selection in visual working memory. Memory \& Cognition, 44(4), 621-632. https://doi.org/10.3758/s13421-015-0584-5

Heuer, A., Schubö, A., \& Crawford, J. D. (2016). Different Cortical Mechanisms for Spatial vs. Feature-Based Attentional Selection in Visual Working Memory. Frontiers in Human Neuroscience, 10. https://doi.org/10.3389/fnhum.2016.00415 JASP Team. (2020). JASP (0.14) [Computer software]. https://jasp-stats.org/ 
Kalogeropoulou, Z., Jagadeesh, A. V., Ohl, S., \& Rolfs, M. (2017). Setting and changing feature priorities in visual short-term memory. Psychonomic Bulletin \& Review, 24(2), 453-458. https://doi.org/10.3758/s13423-016-1094-6

Kondo, A., \& Saiki, J. (2012). Feature-Specific Encoding Flexibility in Visual Working Memory. PLoS ONE, 7(12). https://doi.org/10.1371/journal.pone.0050962

Landman, R., Spekreijse, H., \& Lamme, V. A. F. (2003). Large capacity storage of integrated objects before change blindness. Vision Research, 43(2), 149-164. https://doi.org/10.1016/S0042-6989(02)00402-9

Li, Q., \& Saiki, J. (2015). Different effects of color-based and location-based selection on visual working memory. Attention, Perception, \& Psychophysics, 77(2), 450-463. https://doi.org/10.3758/s13414-014-0775-3

Ling, S., Liu, T., \& Carrasco, M. (2009). How spatial and feature-based attention affect the gain and tuning of population responses. Vision Research, 49(10), 1194-1204. https://doi.org/10.1016/j.visres.2008.05.025

Liu, T., Stevens, S. T., \& Carrasco, M. (2007). Comparing the time course and efficacy of spatial and feature-based attention. Vision Research, 47(1), 108-113. https://doi.org/10.1016/j.visres.2006.09.017

Luck, S. J., \& Vogel, E. K. (2013). Visual working memory capacity: From psychophysics and neurobiology to individual differences. Trends in Cognitive Sciences, 17(8), 391-400. https://doi.org/10.1016/j.tics.2013.06.006

Makovski, T. (2016). Does proactive interference play a significant role in visual working memory tasks? Journal of Experimental Psychology: Learning, Memory, and Cognition, 42(10), 1664-1672. https://doi.org/10.1037/xlm0000262 
Makovski, T., \& Jiang, Y. V. (2007). Distributing versus focusing attention in visual short-term memory. Psychonomic Bulletin \& Review, 14(6), 1072-1078. https://doi.org/10.3758/BF03193093

Makovski, T., \& Jiang, Y. V. (2008). Proactive interference from items previously stored in visual working memory. Memory \& Cognition, 36(1), 43-52. https://doi.org/10.3758/MC.36.1.43

Makovski, T., \& Pertzov, Y. (2015). Attention and memory protection: Interactions between retrospective attention cueing and interference. Quarterly Journal of Experimental Psychology, 68(9), 1735-1743. https://doi.org/10.1080/17470218.2015.1049623

Makovski, T., Sussman, R., \& Jiang, Y. V. (2008). Orienting attention in visual working memory reduces interference from memory probes. Journal of Experimental Psychology: Learning, Memory, and Cognition, 34(2), 369-380. https://doi.org/10.1037/0278-7393.34.2.369

Maljkovic, V., \& Nakayama, K. (1994). Priming of pop-out: I. Role of features. Memory \& Cognition, 22(6), 657-672. Scopus. https://doi.org/10.3758/BF03209251

Maniglia, M. R., \& Souza, A. S. (2020). Age Differences in the Efficiency of Filtering and Ignoring Distraction in Visual Working Memory. Brain Sciences, 10(8), 556. https://doi.org/10.3390/brainsci10080556

Marshall, L., \& Bays, P. M. (2013). Obligatory encoding of task-irrelevant features depletes working memory resources. Journal of Vision, 13(2), 1-13. https://doi.org/10.1167/13.2.21 
Matsukura, M., \& Vecera, S. P. (2015). Selection of multiple cued items is possible during visual short-term memory maintenance. Attention, Perception, \& Psychophysics, 77(5), 1625-1646. https://doi.org/10.3758/s13414-015-0836-2

Maunsell, J. H. R., \& Treue, S. (2006). Feature-based attention in visual cortex. Trends in Neurosciences, 29(6), 317-322. https://doi.org/10.1016/j.tins.2006.04.001

McAdams, C. J., \& Maunsell, J. H. R. (2000). Attention to Both Space and Feature Modulates Neuronal Responses in Macaque Area V4. Journal of Neurophysiology, 83(3), 1751-1755. https://doi.org/10.1152/jn.2000.83.3.1751

Michael, E., Gardelle, V. de, \& Summerfield, C. (2014). Priming by the variability of visual information. Proceedings of the National Academy of Sciences, 111(21), 7873-7878. https://doi.org/10.1073/pnas.1308674111

Morey, R. D., Rouder, J. N., Jamil, T., Forner, K., \& Ly, A. (2018). Package 'bayesfactor'. (0.9.12-4.2) [Computer software]. https://richarddmorey.github.io/BayesFactor/

Murray, A. M., Nobre, A. C., Clark, I. A., Cravo, A. M., \& Stokes, M. G. (2013). Attention Restores Discrete Items to Visual Short-Term Memory. Psychological Science, 24(4), 550-556. https://doi.org/10.1177/0956797612457782

Niklaus, M., Nobre, A. C., \& van Ede, F. (2017). Feature-based attentional weighting and spreading in visual working memory. Scientific Reports, 7(1), 1-10. https://doi.org/10.1038/srep42384

Oberauer, K., \& Eichenberger, S. (2013). Visual working memory declines when more features must be remembered for each object. Memory \& Cognition, 41(8), 12121227. https://doi.org/10.3758/s13421-013-0333-6 
Oberauer, K., \& Lin, H.-Y. (2017). An interference model of visual working memory. Psychological Review, 124(1), 21-59. https://doi.org/10.1037/rev0000044

Oberauer, K., Stoneking, C., Wabersich, D., \& Lin, H.-Y. (2017). Hierarchical Bayesian measurement models for continuous reproduction of visual features from working memory. Journal of Vision, 17(5), 11-11. https://doi.org/10.1167/17.5.11

Park, Y. E., Sy, J. L., Hong, S. W., \& Tong, F. (2017). Reprioritization of Features of Multidimensional Objects Stored in Visual Working Memory. Psychological Science, 28(12), 1773-1785. https://doi.org/10.1177/0956797617719949

Pelli, D. G. (1997). The VideoToolbox software for visual psychophysics: Transforming numbers into movies. Spatial Vision, 10(4), 437-442.

Pertzov, Y., Bays, P. M., Joseph, S., \& Husain, M. (2013). Rapid forgetting prevented by retrospective attention cues. Journal of Experimental Psychology: Human Perception and Performance, 39(5), 1224-1231. https://doi.org/10.1037/a0030947

Pertzov, Y., \& Husain, M. (2014). The privileged role of location in visual working memory. Attention, Perception, \& Psychophysics, 76(7), 1914-1924. https://doi.org/10.3758/s13414-013-0541-y

Pertzov, Y., Manohar, S., \& Husain, M. (2017). Rapid forgetting results from competition over time between items in visual working memory. Journal of Experimental Psychology: Learning, Memory, and Cognition, 43(4), 528-536. https://doi.org/10.1037/xlm0000328

Plummer, M. (2016). JAGS 4.2.0. http://mcmc-jags.sourceforge.net/ 
Poch, C., Capilla, A., Hinojosa, J. A., \& Campo, P. (2017). Selection within working memory based on a color retro-cue modulates alpha oscillations. Neuropsychologia, 106, 133-137. https://doi.org/10.1016/j.neuropsychologia.2017.09.027

Prinzmetal, W., Amiri, H., Allen, K., \& Edwards, T. (1998). Phenomenology of attention: I Color, location, orientation, and spatial frequency. Journal of Experimental Psychology: Human Perception and Performance, 24(1), 261-282. https://doi.org/10.1037/0096-1523.24.1.261

R Core Team. (2018). $R$ : A language and environment for statistical computing. $R$ Foundation for Statistical Computing, Vienna, Austria. https://www.Rproject.org/.

Rerko, L., \& Oberauer, K. (2013). Focused, unfocused, and defocused information in working memory. Journal of Experimental Psychology: Learning, Memory, and Cognition, 39, 1075-1096. https://doi.org/10.1037/a0031172

Rerko, L., Souza, A. S., \& Oberauer, K. (2014). Retro-cue benefits in working memory without sustained focal attention. Memory \& Cognition, 42(5), 712-728. https://doi.org/10.3758/s13421-013-0392-8

Ricker, T. J., \& Hardman, K. O. (2017). The nature of short-term consolidation in visual working memory. Journal of Experimental Psychology: General, 146(11), 15511573. https://doi.org/10.1037/xge0000346

Rouder, J. N. (2014). Optional stopping: No problem for Bayesians. Psychonomic Bulletin \& Review, 21(2), 301-308. https://doi.org/10.3758/s13423-014-0595-4 
Saenz, M., Buracas, G. T., \& Boynton, G. M. (2002). Global effects of feature-based attention in human visual cortex. Nature Neuroscience, 5(7), 631-632. https://doi.org/10.1038/nn876

Schledde, B., Galashan, F. O., Przybyla, M., Kreiter, A. K., \& Wegener, D. (2016). Taskspecific shaping of feature-based attention in area MT. BioRxiv, 058693. https://doi.org/10.1101/058693

Schneegans, S., \& Bays, P. M. (2017). Neural Architecture for Feature Binding in Visual Working Memory. The Journal of Neuroscience, 37(14), 3913-3925. https://doi.org/10.1523/JNEUROSCI.3493-16.2017

Schneegans, S., Harrison, W., \& Bays, P. (2018). Working memory for sequentially presented objects does not rely on location to bind features. Journal of Vision, 18(10), 696. https://doi.org/10.1167/18.10.696

Schneider, D., Mertes, C., \& Wascher, E. (2015). On the fate of non-cued mental representations in visuo-spatial working memory: Evidence by a retro-cuing paradigm. Behavioural Brain Research, 293, 114-124. https://doi.org/10.1016/j.bbr.2015.07.034

Souza, A. S., \& Oberauer, K. (2015). Time-based forgetting in visual working memory reflects temporal distinctiveness, not decay. Psychonomic Bulletin \& Review, 22(1), 156-162. https://doi.org/10.3758/s13423-014-0652-z

Souza, A. S., \& Oberauer, K. (2016). In search of the focus of attention in working memory: 13 years of the retro-cue effect. Attention, Perception, \& Psychophysics, 78(7), 1839-1860. https://doi.org/10.3758/s13414-016-1108-5 
Souza, A. S., \& Oberauer, K. (2017). The contributions of visual and central attention to visual working memory. Attention, Perception, \& Psychophysics, 79(7), 18971916. https://doi.org/10.3758/s13414-017-1357-y

Souza, A. S., Rerko, L., Lin, H.-Y., \& Oberauer, K. (2014). Focused attention improves working memory: Implications for flexible-resource and discrete-capacity models. Attention, Perception, \& Psychophysics, 76(7), 2080-2102. https://doi.org/10.3758/s13414-014-0687-2

Souza, A. S., Rerko, L., \& Oberauer, K. (2014). Unloading and reloading working memory: Attending to one item frees capacity. Journal of Experimental Psychology: Human Perception and Performance, 40(3), 1237-1256. https://doi.org/10.1037/a0036331

Souza, A. S., Rerko, L., \& Oberauer, K. (2015). Refreshing memory traces: Thinking of an item improves retrieval from visual working memory. Annals of the New York Academy of Sciences, 1339(1), 20-31. https://doi.org/10.1111/nyas.12603

Souza, A. S., Rerko, L., \& Oberauer, K. (2016). Getting more from visual working memory: Retro-cues enhance retrieval and protect from visual interference. Journal of Experimental Psychology: Human Perception and Performance, 42(6), 890-910. https://doi.org/10.1037/xhp0000192

Souza, A. S., Thalmann, M., \& Oberauer, K. (2018). The precision of spatial selection into the focus of attention in working memory. Psychonomic Bulletin \& Review, 25(6), 2281-2288. https://doi.org/10.3758/s13423-018-1471-4 
Tabi, Y. A., Husain, M., \& Manohar, S. G. (2019). Recall cues interfere with retrieval from visuospatial working memory. British Journal of Psychology, 110(2), 288305. https://doi.org/10.1111/bjop.12374

Tanoue, R. T., \& Berryhill, M. E. (2012). The mental wormhole: Internal attention shifts without regard for distance. Attention, Perception, \& Psychophysics, 74(6), 11991215. https://doi.org/10.3758/s13414-012-0305-0

Treue, S., \& Trujillo, J. C. M. (1999). Feature-based attention influences motion processing gain in macaque visual cortex. Nature, 399(6736), 575-579. https://doi.org/10.1038/21176

Udale, R., Farrell, S., \& Kent, C. (2018). Task demands determine comparison strategy in whole probe change detection. Journal of Experimental Psychology: Human Perception and Performance, 44(5), 778-796.

https://doi.org/10.1037/xhp0000490

Ueno, T., Allen, R. J., Baddeley, A. D., Hitch, G. J., \& Saito, S. (2011). Disruption of visual feature binding in working memory. Memory \& Cognition, 39(1), 12-23. https://doi.org/10.3758/s13421-010-0013-8

Ueno, T., Mate, J., Allen, R. J., Hitch, G. J., \& Baddeley, A. D. (2011). What goes through the gate? Exploring interference with visual feature binding. Neuropsychologia, 49(6), 1597-1604. https://doi.org/10.1016/j.neuropsychologia.2010.11.030

van Ede, F., Chekroud, S. R., \& Nobre, A. C. (2019). Human gaze tracks attentional focusing in memorized visual space. Nature Human Behaviour. https://doi.org/10.1038/s41562-019-0549-y 
van Moorselaar, D., Olivers, C. N. L., Theeuwes, J., Lamme, V. A. F., \& Sligte, I. G. (2015). Forgotten but not gone: Retro-cue costs and benefits in a double-cueing paradigm suggest multiple states in visual short-term memory. Journal of Experimental Psychology: Learning, Memory, and Cognition, 41(6), 1755-1763. https://doi.org/10.1037/xlm0000124

Wagenmakers, E., Wetzels, R., Borsboom, D., \& van der Maas, H. L. J. (2011). Why psychologists must change the way they analyze their data: The case of psi: Comment on Bem (2011). Journal of Personality and Social Psychology, 100(3), 426-432. https://doi.org/10.1037/a0022790

Wang, D., Kristjansson, A., \& Nakayama, K. (2005). Efficient visual search without topdown or bottom-up guidance. Perception \& Psychophysics, 67(2), 239-253. https://doi.org/10.3758/BF03206488

Wilken, P., \& Ma, W. J. (2004). A detection theory account of change detection. Journal of Vision, 4(12), 1120-1135. https://doi.org/10.1167/4.12.11

Williams, M., \& Woodman, G. F. (2012). Directed forgetting and directed remembering in visual working memory. Journal of Experimental Psychology: Learning, Memory, and Cognition, 38(5), 1206-1220. https://doi.org/10.1037/a0027389

Ye, C., Hu, Z., Ristaniemi, T., Gendron, M., \& Liu, Q. (2016). Retro-dimension-cue benefit in visual working memory. Scientific Reports, 6(1), 35573. https://doi.org/10.1038/srep35573

Zhang, W., \& Luck, S. J. (2008). Discrete fixed-resolution representations in visual working memory. Nature, 453(7192), 233-235. https://doi.org/10.1038/nature06860 
Zhang, W., \& Luck, S. J. (2009). Sudden Death and Gradual Decay in Visual Working Memory. Psychological Science, 20(4), 423-428. https://doi.org/10.1111/j.14679280.2009.02322.x 